\title{
The influence of electronic structure modelling and junction structure on first-principles chiral induced spin selectivity
}

\author{
Martin Sebastian Zöllner, ${ }^{*} \dagger$ Vladimiro Mujica, ${ }^{\ddagger}$ and Carmen Herrmann ${ }^{*} \dagger$ \\ $\dagger$ Department of Chemistry, University of Hamburg, 20146 Hamburg, Germany \\ $\ddagger$ School of Molecular Sciences, Arizona State University, Tempe, Arizona 85287-1604, USA \\ E-mail: martin.zoellner@chemie.uni-hamburg.de; carmen.herrmann@chemie.uni-hamburg.de
}

\begin{abstract}
We have carried out a comprehensive study of the influence of electronic structure modeling and junction structure description on the first-principles calculation of the spin polarization in molecular junctions caused by the chiral induced spin selectivity (CISS) effect. We explore the limits and the sensitivity to modelling decisions of a Landauer / Green's function / density functional theory approach to CISS. We find that although the CISS effect is entirely attributed in the literature to molecular spin filtering, spin-orbit coupling being partially inherited from the metal electrodes plays an important role in our calculations, even though this effect cannot explain the experimental conductance results. Also, an important dependence on the specific description of exchange interaction and spin-orbit coupling is manifest in our approach. This is important because the interplay between exchange effects and spin-orbit coupling may play an important role in the description of the junction magnetic response.
\end{abstract}


Our calculations are relevant for the whole field of spin-polarized electron transport and electron transfer because there is still an open discussion in the literature about the detailed underlying mechanism and the magnitude of relevant physical parameters that need to be included to achieve a consistent description of the CISS effect.

\section{Introduction}

Chiral induced spin selectivity (CISS) is an effect first described in the late 90 s, 1 observed in either the transport or transfer of electrons through chiral molecules or molecular assemblies. The effect translates into the onset of spin polarization, i.e. one of the two spin components has a larger probability of making it through the molecule, depending on its orientation with respect to the transport direction. ${ }^{2}$ This effect has been demonstrated for a variety of molecular systems at room temperature. In addition to its importance for fundamental science, the CISS effect has large potential for applications such as spin-dependent chemistry, electrochemical water splitting, ${ }^{3 / 6}$ enantiomer separation with achiral magnetic substrates, ${ }^{7}$ and spintronic devices. ${ }^{2 / 8}$ It may also contribute to the large efficiency of electron transfer in biological systems due to the coupling of spin angular momentum and linear momentum reducing backscattering. 219

The CISS effect has been observed in tunneling junctions, 10 in photoemission through chiral

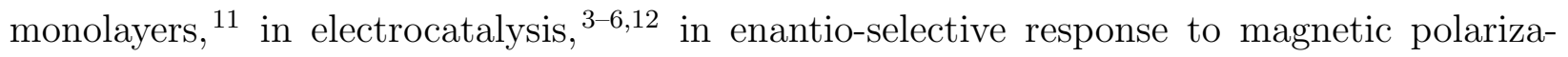
tion, $\frac{13}{}$ and in magnetism-related properties induced in a conventional superconductor ${ }^{14}$ for a variety of molecules ranging from DNA, ${ }^{15]}$ peptides $^{10[18}$ and proteins ${ }^{19}$ to helicenes..$^{20 \mid 21}$ Most, but not all of these systems are helical. While most experiments were done on molecular ensembles, the effect has been shown in single-molecule junctions. 10 Despite initial assumptions that the SOC of the substrate (gold) might play a role, substantial CISS has been observed in photoemission on substrates with small SOC (aluminium and copper). 1920 
In conductance measurements, spin polarizations of up to $60 \%$ were obtained for a single 22 -amino-acid $\alpha$-helical peptide on a gold substrate contacted by a nickel tip.10 This is in line with a difference in single-molecule current of roughly one order of magnitude between electrons of different spin orientations in conductance experiments on 14-amino-acide peptide monolayers on nickel, contacted via gold nanoparticles,, 10 and with the substantial spin polarizations measured for molecular systems in various other experimental configurations. ${ }^{2}$ A theoretical description of CISS requires taking spin-orbit coupling (SOC) into account. While tight-binding and first-principles theoretical descriptions of a related effect, spin splitting in surface states of $\mathrm{Au}(111)$ surfaces induced by Rashba SOC, 22 can yield quantitative accuracy, ${ }^{2324}$ such tight-binding ${ }^{25 / 35}$ and first-principles ${ }^{36137}$ descriptions of the CISS effect so far underestimate it by several orders of magnitude. ${ }^{38(39)}$ It has been suggested that this discrepancy is due to dephasing $25 \sqrt{259}$ or local leakages, ${ }^{35}$ which can be modeled in terms of Büttiker probes. ${ }^{40}+45$ There is also substantial debate in the current literature as to whether CISS can be observed in two-terminal configurations at all. $\stackrel{46+49}{46}$ Other proposed explanations include nonmagnetic electrodes becoming spin-polarized in a nonequilibrium situation due to the presence of the chiral molecule,, 50 charge transfer between molecule and substrate,, 51 and electron correlation in nonequilibrium playing a substantial role. $\frac{52}{5}$

A first-principles-based description of CISS is essential for understanding subtle aspects of structure-property relationships, and for predictive simulations. Deciding which of the mentioned suggestions is (or are) essential for a reliable first-principles description of CISS is a formidable task. It is further complicated by the fact that even for phenomena where the basic physical mechanisms are known, practically feasible approaches such as KohnSham density functional theory (KS-DFT) may yield quantitatively and sometimes even qualitatively wrong results with an inappropriate choice of the approximate exchangecorrelation functional. ${ }^{53154}$ Particularly challenging for KS-DFT, both from practical and formal perspectives, are spin-related properties such as spin crossover and exchange spin 
coupling. $53 \sqrt[55]{55}$ There are also formal issues when combining KS-DFT with nonequilibrium phenomena. $\stackrel{39 \mid 56}{59}$ Nonetheless, KS-DFT has proven very successful in understanding singlemolecule conductance. 3915961 Absolute conductance values from the experiment are hard to reproduce because of issues such as structural fluctuations and level alignment, $\stackrel{59}{\text { but trends }}$ and qualitative properties are described very well in many cases, and it is possible to get the right order of magnitude for conductance. ${ }^{61}$ This is in contrast to CISS, where presentday first-principles (and tight-binding) results are consistently lower than the experimentally required values, by several orders of magnitude.

In addition to future experiments and further developments of theoretical models, statistical analyses of errors and uncertainty quantification ${ }^{62 / 72}$ and machine-learning approaches 73 may help establishing a reliable first-principles theory of CISS. As a first step, we need to identify how first-principles modeling decisions affect central quantities relevant for CISS. This is interesting and necessary even before a full first-principles theory has been established, to prevent identifying a certain theoretical approach as quantitatively correct due to error compensation later on. To this end, we build on our previous work, ${ }^{37}$ in which we had identified the imaginary part of the effective single-particle Hamiltonian matrix (in HartreeFock theory or KS-DFT: the Fock matrix) as responsible for SOC-induced spin polarization in closed-shell systems within the Landauer approach. This approach describes the correct

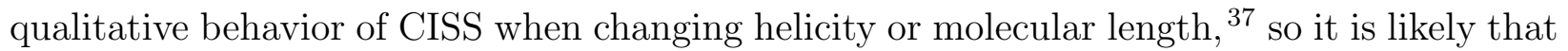
it plays a part in modeling CISS (with a yet unknown amplification mechanism missing, as discussed above).

We first analyze the influence of metal clusters modeling the electrodes by changing their shape, size, and material $(\mathrm{Cu}, \mathrm{Ag}, \mathrm{Au})$ for the same ideal carbon helix as studied in our previous work,,$\frac{37}{}$ followed by an analysis of the effect of going from equidistant to alternately spaced carbon atoms in the helix. This is important since parts of the SOC may be inherited by the electrode, and this inheritance may be affected by the electronic and atomistic 
structures of both helix and electrode. We then look into the dependence of the quantities entering spin polarization in CISS on modeling decisions such as the admixture of exact exchange in the approximate exchange-correlation functional. This is important since the imaginary part of the effective single-particle Hamiltonian matrix (in a framework including SOC) depends on it. $\frac{79 \mid 80}{7 n a l l y, ~ w e ~ d o ~ a ~ r e p r e s e n t a t i v e ~ c h e c k ~ o f ~ h o w ~ a ~ o u r ~ f i n d i n g s ~ f o r ~ a n ~}$ artificial system transfer to realistic systems, by varying the exact exchange admixture for the spin polarization in a helicene derivative.

\section{Theoretical background}

In the vast majority of theoretical studies, it is assumed that spin-orbit coupling plays a decisive role for understanding CISS (even though it has been suggested that it is possible to induce spin-polarized currents in the absence of SOC in helical systems ${ }^{81}$ ). This is consistent with the fact that for curved carbon systems, SOC is roughly three orders of magnitude larger than for flat graphene ${ }^{82}$ (where it is around a few $\mu \mathrm{eV}^{85186}$ ).

\subsection{Spin-orbit coupling}

For a single electron moving within the potential of a nucleus, spin-orbit coupling is the interaction of the electron's spin with its motion in the nuclear potential. This type of interaction can be described by the Breit-Pauli Hamiltonian, $\stackrel{87}{ }$ where the one-electron SOC is given as

$$
\hat{H}^{\mathrm{BP}, \text { so }}=\frac{1}{2 m_{e}^{2} c^{2}} \sum_{i} \sum_{I} \frac{Z_{I}\left(\boldsymbol{r}_{i I} \times \hat{\boldsymbol{p}}_{i}\right) \cdot \hat{\boldsymbol{s}}_{i}}{r_{i I}^{3}},
$$

where $m_{e}$ is the mass of the electron, $c$ is the speed of light, $Z_{I}$ is the charge number of nucleus $I, \hat{\boldsymbol{p}}_{i}$ is the momentum operator of electron $i, \hat{\boldsymbol{s}}_{i}$ is the spin operator of electron $i$, 
and $r_{i I}$ is the distance between nucleus $I$ and electron $i$.

In many-electron systems, additional spin-orbit coupling terms arise due to the potential and motion of the other electrons. Those two-electron SOC terms can be split into two contributions, the spin-same-orbit coupling,

$$
\hat{H}^{\mathrm{BP}, \mathrm{sso}}=-\frac{1}{2 m_{e}^{2} c^{2}} \sum_{i, j \neq i} \frac{\left(\boldsymbol{r}_{i j} \times \hat{\boldsymbol{p}}_{i}\right) \cdot \hat{\boldsymbol{s}}_{i}}{r_{i j}^{3}}
$$

and the spin-other-orbit coupling,

$$
\hat{H}^{\mathrm{BP}, \text { soo }}=-\frac{1}{m_{e}^{2} c^{2}} \sum_{i, j \neq i} \frac{\left(\boldsymbol{r}_{i j} \times \hat{\boldsymbol{p}}_{i}\right) \cdot \hat{\boldsymbol{s}}_{j}}{r_{i j}^{3}} .
$$

$\boldsymbol{r}_{i j}$ is the operator corresponding to the distance between electron $i$ and electron $j$.

In many KS-DFT calculations, two-electron SOC is neglected ${ }^{1}$,

\section{$2.2 \quad$ Electron transport considering spin-orbit coupling}

To estimate the CISS effect based on KS-DFT electronic structure calculations, we employ the Landauer-Imry-Büttiker approach, ${ }^{89}$ which assumes coherent tunneling as the transport mechanism. This is a good assumption for the short oligopeptides for which CISS has been studied experimentally,, 90191 and for helicen $£^{2}$.

When combined with first-principles electronic structure methods relying on an effective single-particle picture, the Landauer-Imry-Büttiker approach is based on a partitioning scheme, ${ }^{93}$ where the effective single-particle Hamiltonian matrix (in the following shortly

\footnotetext{
${ }^{1}$ Parts of the two-electron SOC can, for instance, be approximated in a one-electron formulation by using the Kohn-Sham potential for the calculation of the SOC instead of the bare nuclear potential. $\underline{\text { 88 }}$

${ }^{2}$ The description of transport for longer molecules, e.g segments of DNA, ${ }^{92}$ requires the inclusion of incoherent transport.
} 
called Hamiltonian matrix) of the complete junction is partitioned as

$$
\boldsymbol{H}_{t o t}=\left(\begin{array}{ccc}
\boldsymbol{H}_{L} & \boldsymbol{V}_{L C} & \mathbf{0} \\
\boldsymbol{V}_{C L} & \boldsymbol{H}_{C} & \boldsymbol{V}_{C R} \\
\mathbf{0} & \boldsymbol{V}_{R C} & \boldsymbol{H}_{R}
\end{array}\right)
$$

$\boldsymbol{H}_{L}$ is the Hamiltonian matrix of the left electrode, $\boldsymbol{H}_{R}$ the Hamiltonian matrix of the right electrode, $\boldsymbol{H}_{C}$ the Hamiltonian matrix of the central region, and $\boldsymbol{V}_{X Y}$ describes the coupling of region $X$ with region $Y$. This partitioning requires single-particle basis functions which are (at least mainly) localized in the respective regions, as is the case for the popular atom-centered Gaussian basis functions. To estimate the CISS effect, we use an approach described, among others, in Refs. ${ }^{94 \mid 95}$ If SOC is considered in DFT, single-electron quantities become two-component spinors, and all matrices assume the form

$$
\mathbf{M}=\left(\begin{array}{cc}
\mathbf{M}^{\uparrow \uparrow} & \mathbf{M}^{\uparrow \downarrow} \\
\mathbf{M}^{\downarrow \uparrow} & \mathbf{M}^{\downarrow \downarrow}
\end{array}\right)
$$

The tunneling current for electrons originating with spin orientation $\sigma$ from the right electrode and arriving with spin orientation $\sigma^{\prime}$ at the left electrode $\left(\sigma, \sigma^{\prime} \in\{\uparrow, \downarrow\}\right)$ can be calculated as

$$
I_{\sigma, \sigma^{\prime}}=\frac{e}{h} \int_{E-\frac{e V}{2}}^{E+\frac{e V}{2}} T^{\sigma, \sigma^{\prime}}(E) d E
$$

where $E$ is the energy, $V$ is the applied bias voltage, which is assumed to drop symmetrically in the two junction electrode, and $T$ is the transmission function. The transmission function roughly describes the probability of an electron originating with spin orientation $\sigma$ and arriving with spin orientation $\sigma^{\prime}$ to tunnel through the central region from right to left. It 
can be calculated as

$$
T^{\sigma, \sigma^{\prime}}=\operatorname{Tr}\left[\boldsymbol{\Gamma}_{L}^{\sigma^{\prime} \sigma^{\prime}} \mathbf{G}^{\sigma \sigma^{\prime}} \boldsymbol{\Gamma}_{R}^{\sigma \sigma}\left(\mathbf{G}^{\sigma \sigma^{\prime}}\right)^{\dagger}\right]
$$

where $\Gamma_{L}$ and $\Gamma_{L}$ are the so called coupling matrices of the left and the right electrode and $\boldsymbol{G}$ is the Green's function of the central region. The coupling matrices describe the interaction of the electrodes with the central region. The energy dependence of $T$ and of the matrices on the right has been dropped here for readability. The transmission at the Fermi energy $E_{F}$ allows estimating the zero-bias conductance,

$$
g_{\sigma, \sigma^{\prime}}(0 \mathrm{~V})=\frac{e}{h} T^{\sigma, \sigma^{\prime}}\left(E_{F}\right)
$$

The coupling matrices will be assumed as energy-independent here (see discussion on the wide-band limit in Section 3), while the Green's functions are energy-dependent quantities evaluated as

$$
\mathbf{G}^{\sigma, \sigma^{\prime}}=\frac{1}{E \mathbf{S}_{C}^{\sigma, \sigma^{\prime}}-\mathbf{H}_{C}^{\sigma, \sigma^{\prime}}-\boldsymbol{\Sigma}_{L}^{\sigma, \sigma^{\prime}}-\boldsymbol{\Sigma}_{R}^{\sigma, \sigma^{\prime}}},
$$

where $\boldsymbol{S}_{C}$ is the overlap matrix for the atom-centered basis functions (which has all elements equal to zero for $\sigma \neq \sigma^{\prime}$ ) and $\mathbf{H}_{C}^{\sigma, \sigma^{\prime}}$ is the Hamiltonian matrix of the central region. $\boldsymbol{\Sigma}_{L}^{\sigma, \sigma^{\prime}}$ and $\Sigma_{L}^{\sigma, \sigma^{\prime}}$ are the self-energies which describe the effect of the left and right electrode, respectively, on the central region,

$$
\boldsymbol{\Sigma}_{X}^{\sigma, \sigma^{\prime}}=\Delta_{X}^{\sigma, \sigma^{\prime}}-\frac{i}{2} \boldsymbol{\Gamma}_{X}^{\sigma, \sigma^{\prime}}
$$

Here, we assume that the real part of the self-energies (which leads to a shift of the centralsubsystem effective single-particle levels) is already included in $\mathbf{H}_{C}$, thus setting $\boldsymbol{\Delta}_{X}^{\sigma, \sigma^{\prime}}$ to zero. 
If SOC is neglected within the electrodes, the spin-flip blocks of $\boldsymbol{\Gamma}_{X}$ and $\boldsymbol{\Sigma}_{X}$ become zero and the spin within the electrodes remains well defined. This approximation allows to define an overall transmission as the sum of $T$ four spin-dependent contributions,

$$
2 T=T^{\uparrow \uparrow}+T^{\downarrow \uparrow}+T^{\uparrow \downarrow}+T^{\downarrow \downarrow}
$$

where the first and last terms describe spin-conserving transmissions, while the second and third terms describe spin-flip transmissions. The factor of 2 takes into account that for closed-shell systems without SOC, $T$ is usually interpreted as the probability of an electron of a given spin orientation to tunnel through the junction, whereas here terms for both spin orientations are added un

In the experiment, measuring the spin polarization $P_{S}$ of the transmitted electrons requires a magnetic electrode. ${ }^{10}$ In our calculations, we can mimick measurement by an ideal magnetic electrode by employing a closed-shell one like gold, since we have access to all four terms in Eq. (11) and can thus evaluate $P_{S}$ as the normalized difference between transmission for all spin-up electrons and all spin-down electrons making it to the other side,

$$
P=\frac{T^{\uparrow \uparrow}+T^{\downarrow \uparrow}-T^{\uparrow \downarrow}-T^{\downarrow \downarrow}}{2 T} .
$$

Again, this is an energy-dependent quantity, and an estimate for the polarization at zero bias can be obtained by considering $P\left(E_{F}\right)$.

Note that all Hamiltonian matrix elements are obtained from a standard self-consistent field algorithm on a finite cluster-molecule-cluster system (see below) in equilibrium. This is a good approximation for transport properties close to zero bias, but it may miss strongly nonlinear effects which may play a role for larger bias voltages.

\footnotetext{
${ }^{3}$ In Ref., ${ }^{37}$ the factor of 2 was omitted, i.e., $T$ as defined here and in most of the literature is equal to $1 / 2 T$ as defined in Ref. ${ }^{37}$ This distinction does not affect the values of the property crucial for CISS, the polarization $P_{S}$.
} 


\section{Methods}

We calculated $P_{S}$ for three different systems: perfect carbon helixes with evenly and alternatingly spaced atoms, respectively, and a helicene derivative as studied prevously in the experiment. Cartesian coordinates of all structures are provided in the Supporting Information.

\subsection{Construction of junctions of the perfect helical systems}

The ideal helical structures were constructed as in Ref.: 37 carbon atoms were placed along a perfect right-handed helical path (radius $=2 \AA$, pitch $=3 \AA$ ), either evenly spaced $(1.3 \AA$ ) or alternately spaced (1.2 $\AA 1.3 \AA$ ). Two hydrogen atoms were attached to each end, whose positions were optimized while keeping the carbon atoms fixed. The gold-moleculegold junctions were built by placing the terminal carbon atoms in the fcc-position between two metal clusters with a carbon-metal distance $d_{\mathrm{C}-\text { Metal }}$ of $2.48 \AA$ (see Figure 1 ).

Several metal clusters were studied, differing in size and the type of metal. For all clusters, the experimental $d_{\mathrm{Au}-\mathrm{Au}}$ distance of $2.88 \AA$ was used for comparability. Three different metals were considered $(\mathrm{Au}, \mathrm{Cu}, \mathrm{Ag})$ to investigate the amount of inherited SOC from the metal. To check the dependence on the size of the metal cluster, four different cluster sizes were used, namely 6-3-1, 16-0-0, 16-16-0, and 16-16-16 (where the numbers indicate the number of metal atoms in subsequent layers, see Figure 2]. 

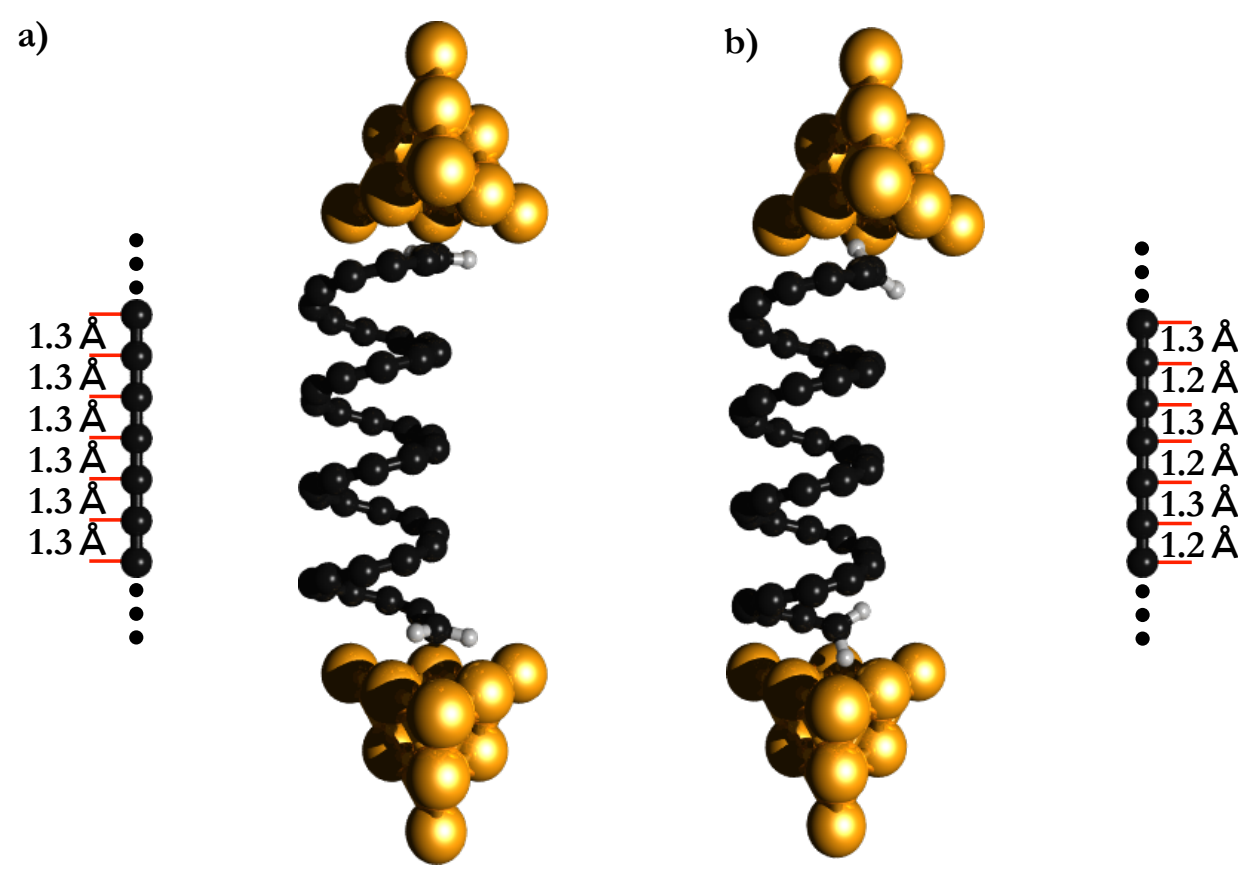

Figure 1: Structures of the gold-molecule-gold junction of the carbon helix with a) equal and b) alternating bond distances.

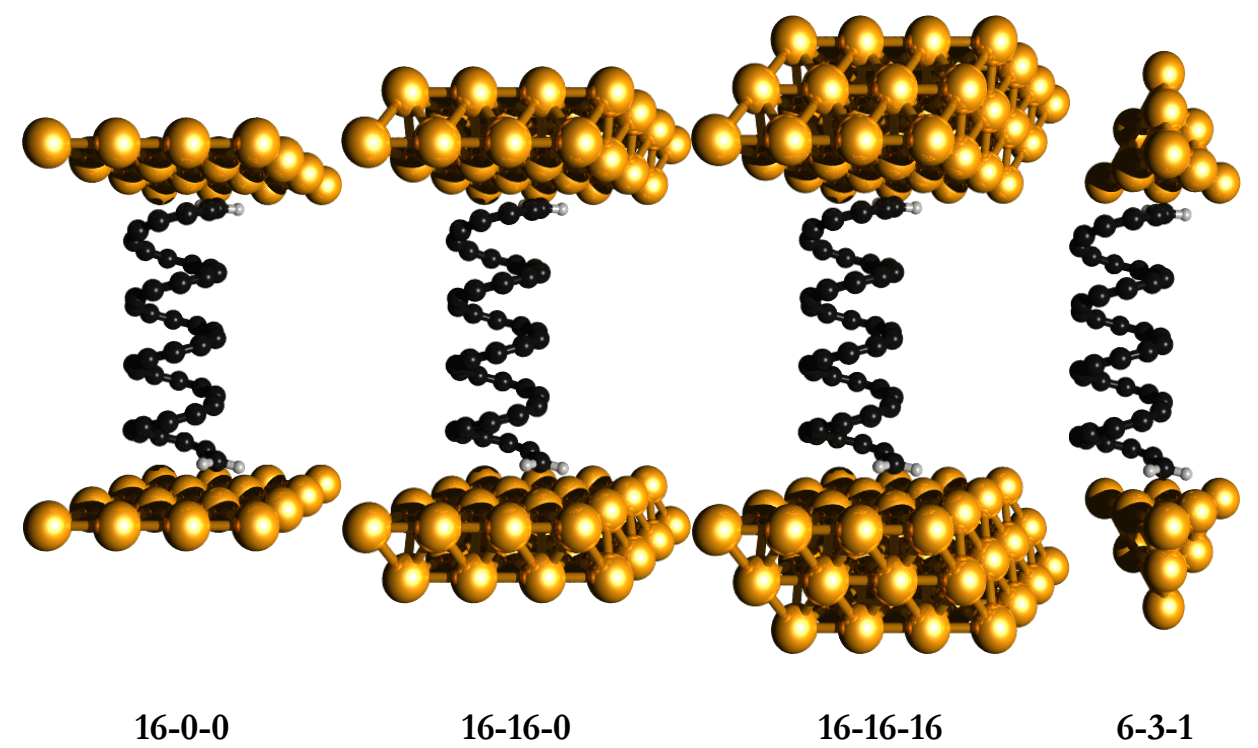

Figure 2: Structures of the gold-molecule-gold junction for gold clusters of different sizes and shapes to simulate the electrodes. The clusters are named by the number of atoms in each layer. 


\subsection{Construction of junctions of the helicene molecule}

As an experimental system, a cationic helicene derivative was studied, for which a spinpolarization of roughly $50 \%$ was measured for a monolayer on highly oriented pyrolytic graphite with a magnetized iron-coated Si tip in the conductive atomic force microscope. ${ }^{21}$ The structure was optimized without the counter anion in vacuum, and the junction was built by placing the optimized helicene cation between two 20-atomic gold clusters (see Figure 3). The molecule-gold distance was estimated based on a DFT-calculated gold-benzene distance of $3.1 \AA .96$

a)

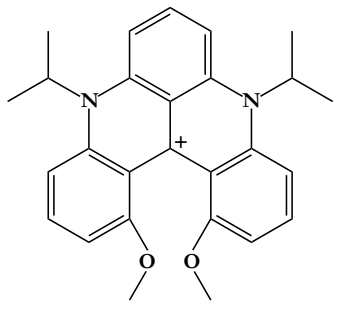

b)

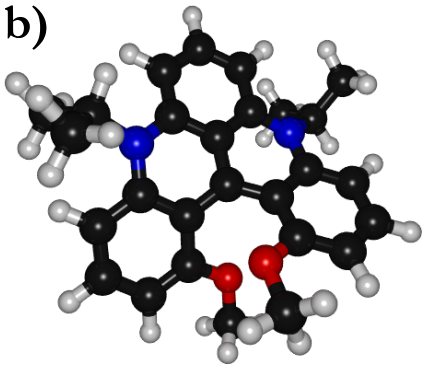

Isolated

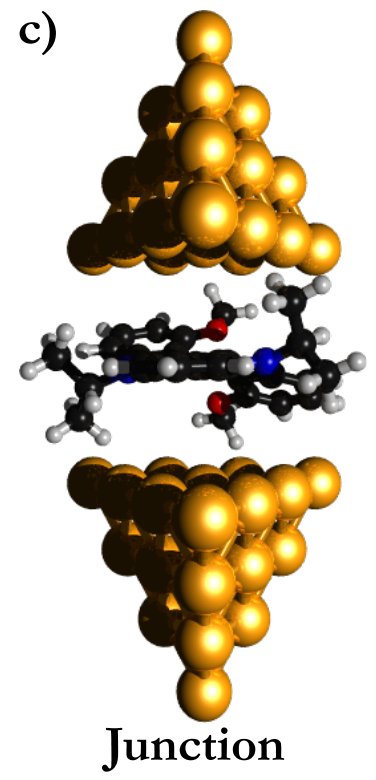

Figure 3: a) Lewis structure of the investigated helicene cation, b) optimized structure of the cation and c) gold-helicene-gold junction.

\subsection{Computational settings}

TuRBomole $7.1^{97-100}$ was employed for the optimizations of all structures. The generalizedgradient approximation (GGA) exchange-correlation functional introduced by Perdew, Burke and Ernzerhof $(\mathrm{PBE})^{101102}$ was used, in combination with the def2-TZVP ${ }^{1031104}$ basis set. Dispersion interactions were taken into account by Grimme's dispersion correction DFT- 
D3 ${ }^{105}$ including Becke-Johnson damping. ${ }^{106}$ To speed up the calculations, the resolution-ofthe-identity method ${ }^{107108}$ with the corresponding auxiliary basis set ${ }^{109}$ was employed. The structures were allowed to relax until the change of energy was below $10^{-6}$ a.u. and the gradient was below $10^{-4}$ a.u.. The energy convergence threshold within the self-consistent field algorithm was set to $10^{-7}$ a.u..

For the calculations of the transmission functions, several different methodologies (see Table (1) were applied: relativistic effects were taken into account by using the exact twocomponent (X2C) method ${ }^{1101111}$ and the $\mathrm{x} 2 \mathrm{c}-\mathrm{SVPall}-2 \mathrm{c}^{[112}$ basis set (Turbomole), the zeroth-order regular approximation (ZORA) $)^{113 \cdot 117}$ and the ZORA-DZ ${ }^{118}$ basis set (ADF ${ }^{119}[121)$, or effective-core potentials using the dhf-SVP ${ }^{[122]}$ basis set (TURBOmOLE). Additionally, calculations with the Dirac ${ }^{123}$ code in combination with the dyall.ae2z $\mathrm{z}^{[124}$ basis set and the $\mathrm{X} 2 \mathrm{C}$ method were done. For all calculations, the exchange-correlation energy was calculated based on the B3LYP $125+127$ functional.

For each system, two electronic-structure calculations were carried out: First a one-component KS-DFT calculation without taking SOC into account, then a two-component KS-DFT calculation including spin-orbit coupling (using the molecular orbitals from the one-component calculation as an initial guess). The one-component and two-component Hamiltonian and overlap matrices were extracted from the output files of the employed DFT programs. These matrices were then postprocessed as described in the Theory section employing our program package Artaios. $\frac{128}{128}$ The self-energies $\boldsymbol{\Sigma}_{X}$ for the electrodes $X \in\{L, R\}$ were created from the matrices of the one-component calculation,

$$
\boldsymbol{\Sigma}_{X}=\left(E \mathbf{S}_{X C}-\mathbf{H}_{X C}\right)^{\dagger} \mathbf{g}_{X}\left(E \mathbf{S}_{X C}-\mathbf{H}_{X C}\right) .
$$

Here, $\mathbf{S}_{X C}$ and $\mathbf{H}_{X C}$ denote the overlap and Hamiltonian matrix blocks containing basis functions on both the central region and the electrode $X$, and $\mathbf{g}_{X}$ is the Green's function of the electrode $X$. The Green's function is approximated within the wide-band limit, 1229 
assuming a constant local density of states LDOS $^{\text {const }}$

$$
\left(\mathbf{g}_{X}\right)_{i j}=-\mathrm{i} \pi \operatorname{LDOS}^{\text {const }} \delta_{i j}
$$

The value for the constant LDOS was set to $0.036 \mathrm{eV}^{-1}$ as calculated by DFT for the $6 s$ band of gold $\frac{130}{130}$ This is a reasonable assumption for gold with its flat LDOS around the Fermi energy. The Green's function of the central region in the presence of the electrodes was calculated as

$$
\mathbf{G}=\frac{1}{E \mathbf{S}_{C}-\mathbf{H}_{C}-\Sigma_{L}-\Sigma_{R}}
$$

$\mathbf{H}_{C}$ and $\mathbf{S}_{C}$ were extracted from the respective matrices of the two-component calculation.

Transmission functions and spin polarizations are reported with respect to an estimated Fermi energy of $E_{F}=-5 \mathrm{eV}$ for gold. Since we did not use the experimental $d_{\mathrm{Ag}-\mathrm{Ag}} / d_{\mathrm{Cu}-\mathrm{Cu}}$ distance, we also show the transmission functions calculated with copper and silver clusters shifted against the estimated Fermi energy of gold.

This postprocessing methododology neglects nonequilibrium effects on the electronic structure. For small bias voltages, this is likely a good approximation. ${ }^{131132}$ However, it cannot be excluded that for a quantitative description of CISS, including such nonequilibrium effects explicitly may play an important role (also see the discussion in the conclusion).

Table 1: KS-DFT methodological choices for the calculation of transmission functions in this work. All spin-orbit coupling calculations with TURBOMOLE were done using the "\$kramers" and "\$coulex" keywords.

\begin{tabular}{c|c|c|c|c}
\hline \hline Methodology & DFT code & Functional & Relativistic effects & basis set \\
\hline A & TURBOMOLE 7.1 & B3LYP & X2C & x2c-SVPall-2c \\
B & TURBOMOLE 7.1 & B3LYP & effective-core potential & dhf-SVP \\
C & DirAC & B3LYP & X2C & dyall.ae2z \\
D & ADF & B3LYP & ZORA & ZORA-DZ \\
\hline \hline
\end{tabular}




\section{Dependence of CISS on structural parameters}

\subsection{Electrode cluster composition}

To investigate to what extent the polarization $P_{S}$ for the perfect helical system originates from intrinsic SOC of the helix or from SOC inherited from the gold clusters, we have calculated $P_{S}$ for three junctions: $\mathrm{Au}-\mathrm{helix}-\mathrm{Au}, \mathrm{Ag}-\mathrm{helix}-\mathrm{Ag}$, and $\mathrm{Cu}-\mathrm{helix}-\mathrm{Cu}$, employing methodology A (see Table 1). For all three electrodes, the interatomic distance of bulk gold $(2.88 \AA)$ was used for comparability.

$P_{S}$ massively decreases with the atomic number of the element which is used to build the electrode (see Figure 4). This trend can also be observed in $\operatorname{Im}\left(\mathbf{H}_{C}\right)$. This reinforces our earlier finding of SOC being inherited to a substantial degree from the electrodes for the $\mathrm{Au}-$ helix-Au system, ${ }^{37}$ similar to proximity effects in graphene on gold. ${ }^{1331134}$ In contrast, at least in photoemission experiments, a substantial spin polarization resulting from CISS is observed for light-element substrates just as much as for gold.19120 This suggests that molecular conductance experiments on CISS employing electrodes with less intrinsic SOC would be beneficial to check whether CISS is equally independent of substrate SOC in this type of experiment as in photoemission, and that as for as long as this is not settled, to keep in mind that first-principles CISS results on molecular conductance may be affected by a potentially artificial SOC transfer from the substrate. However, these results do not indicate that CISS as obtained from the Landauer + two-component DFT approach as employed here

only reflects substrate SOC: As shown in Ref., ${ }^{37}$ for the same set of gold clusters, going to a longer helix increases polarization considerably, in agreement with experimental results. $\frac{135}{13}$ To gain further insight into this phenomenon, we will study in Section 4.3 whether the amount of SOC transfer is linked to the near-metallic properties of our model helix resulting from the equidistant spacing of the carbon atoms. 

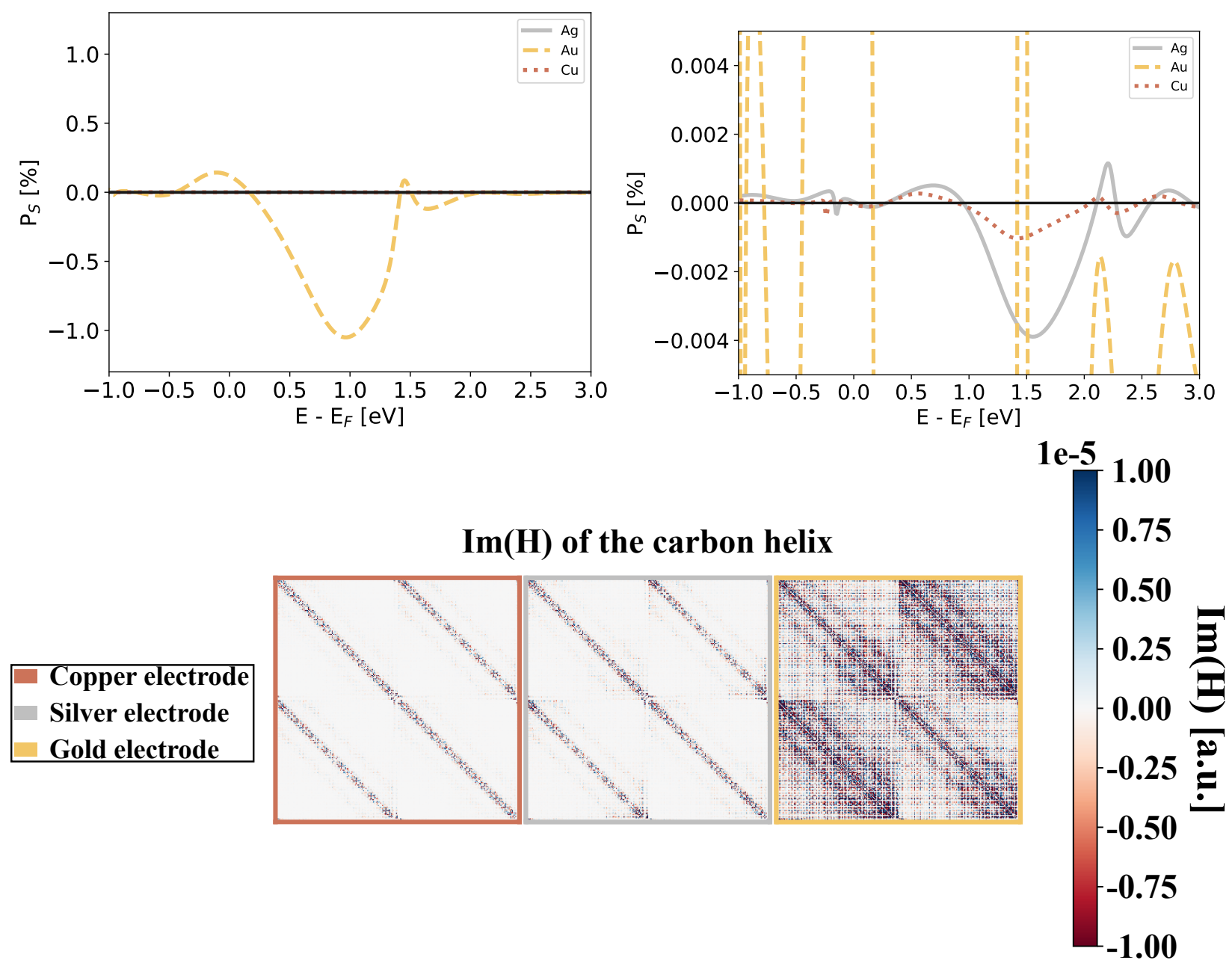

Figure 4: B3LYP/x2c-SVPall-2c spin polarization $P_{S}$ of the transmitted electrons for the equidistant carbon helix junction with 10-atom (6-3-1) metal clusters, using different metal atoms to build the electrodes $(\mathrm{Cu}, \mathrm{Ag}, \mathrm{Au})$. While for gold electrodes, $P_{S}$ is about $1 \%$, it is negligibly small for copper and silver electrodes. This is in agreement with $\operatorname{Im}\left(\mathbf{H}_{C}\right)$, which is much larger for gold compared with copper or silver as electrode material.

\subsection{Electrode cluster size and effective core potentials}

Contact effects have been studied previously in a tight-binding model, 136 and it has been pointed out in several theoretical $\frac{45[136}{4}$ studies that one-dimensional leads prevent spin polarization through CISS. It has even been suggested that a suitably chosen connection to the electrodes can lead to spin filtering in achiral systems. ${ }^{137}$ If $P_{S}$ is mainly induced by the SOC inherited from the gold atoms, two other parameters are expected to be important for 
the calculation of $P_{S}$ : the size and the shape of the clusters. This may influence the coupling of the electrode with the helix and thus the inherited SOC. Since all-electron calculations using large gold clusters are expensive, we used methodology B here (see Table 1), i.e., fullyrelativistic effective core potentials which only take into account the SOC of gold. This is justified, since the results from the previous section clearly indicate that the inherited SOC from the gold is most important for $P_{S}$, and because the calculations for the ten-atomic gold clusters with effective-core potentials reproduce the results from the all-electron calculation quite well (see Figure 5).

The calculations for different gold clusters as electrodes (Figure 2) clearly show that $P_{S}$ depends on the cluster shape and size (see Figure 5). $P_{S}$ calculated with mono- and a bilayers of a $4 \times 4$ gold sheet is negligibly small. Adding a third layer increases $P_{S}$ to the order of $0.1 \%$, but it remains much lower than $P_{S}$ for the original ten-atom gold cluster (built from three layers as 6-3-1). This suggests that there may be counteracting effects at play: an increase of SOC transfer when adding a third layer, and a decrease of this transfer when extending the cluster sideways. These results suggest a need of converging $P_{S}$ with respect to cluster size. However, two-component calculations in general and allelectron calculations using the $\mathrm{X} 2 \mathrm{C}$ decoupling scheme in particular are expensive, making such studies computationally demanding. It may be that going to a description employing semiinfinite electrodes under periodic boundary conditions may alleviate the shape and size dependence somewhat, but our studies suggest that this is something that should be checked carefully, at least if SOC transfer from the electrodes plays a role. 


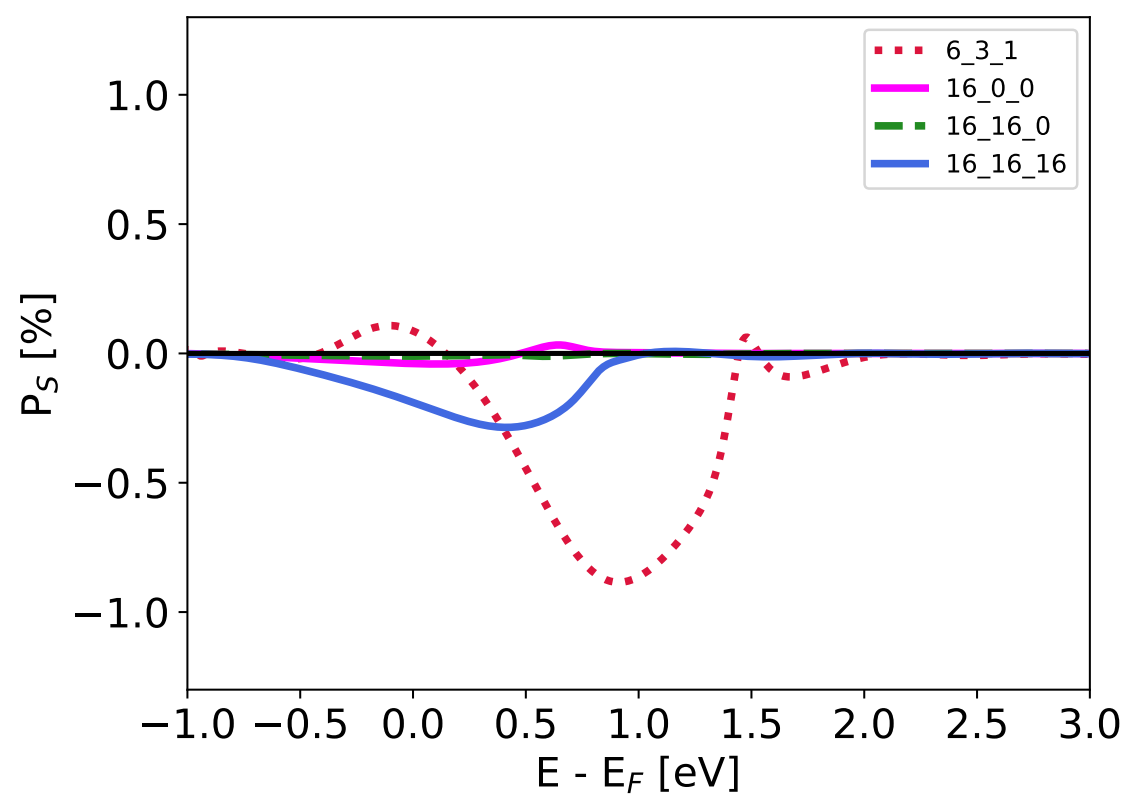

Figure 5: Calculated (B3LYP/dhf-SVP) spin polarization of the transmitted electrons $\left(P_{S}\right)$ through the equidistant helix connected to gold clusters of different sizes and shapes. $P_{S}$ strongly depends on the gold cluster. With $4 \times 4$ gold layers, $P_{S}$ increases with the number of layers (monolayer $=16 \_0 \_0$, bilayer $=16 \_16 \_0$, trilayer $\left.=16 \_16 \_16\right)$. However, if a smaller three-layered gold-cluster is employed (first layer six gold atoms, second layer three gold atoms, third layer one gold atom = $\left.6 \_3 \_1\right), P_{S}$ is much larger than for the 16_16_16 cluster.
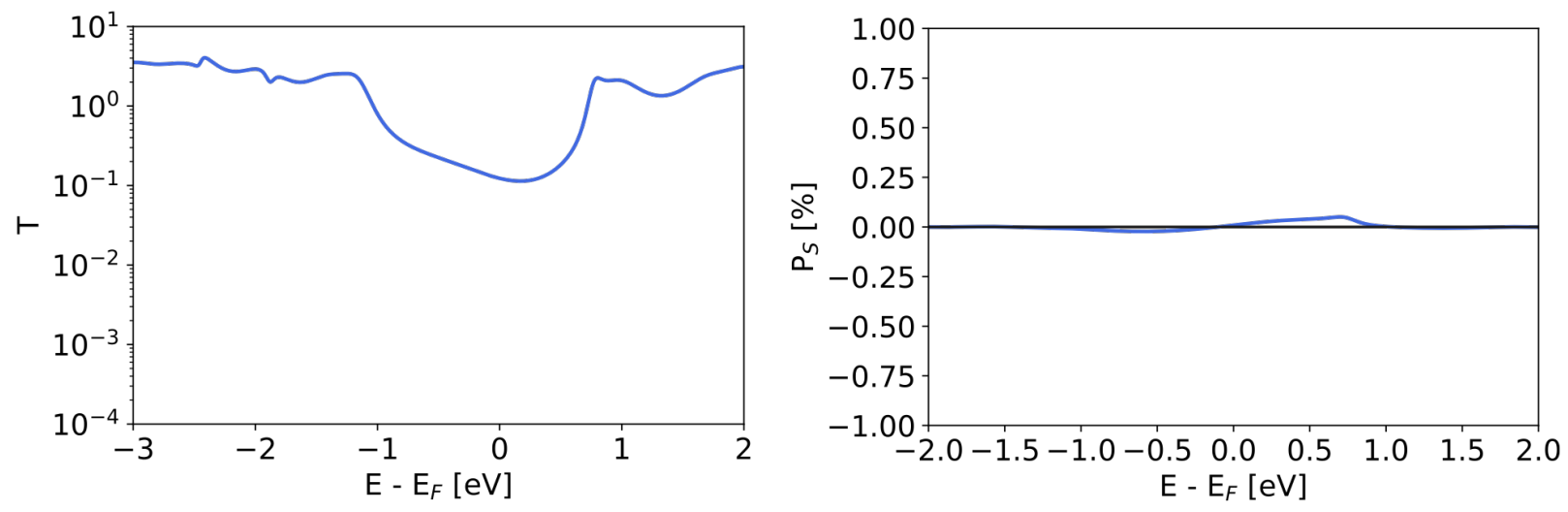

Figure 6: Transmission function and $P_{S}$ calculated for the perfect helix with alternating bond pattern. In contrast to the equidistant helix, even for $20 \%$ exact exchange admixture, a dip occurs in the transmission function, indicating a sizeable HOMO-LUMO gap for the alternating system in comparison with the equidistant system. $P_{S}$ is much smaller than for the equidistant structure. 


\subsection{Carbon helix with alternating bond pattern}

The perfect helix with equidistant carbon-carbon distances resembles a metallic system, which makes a direct transfer to experimental systems difficult. Thus, we also calculated a carbon helix with an alternating bond pattern, which corresponds better to a system with a band gap, such as stable molecular helices.

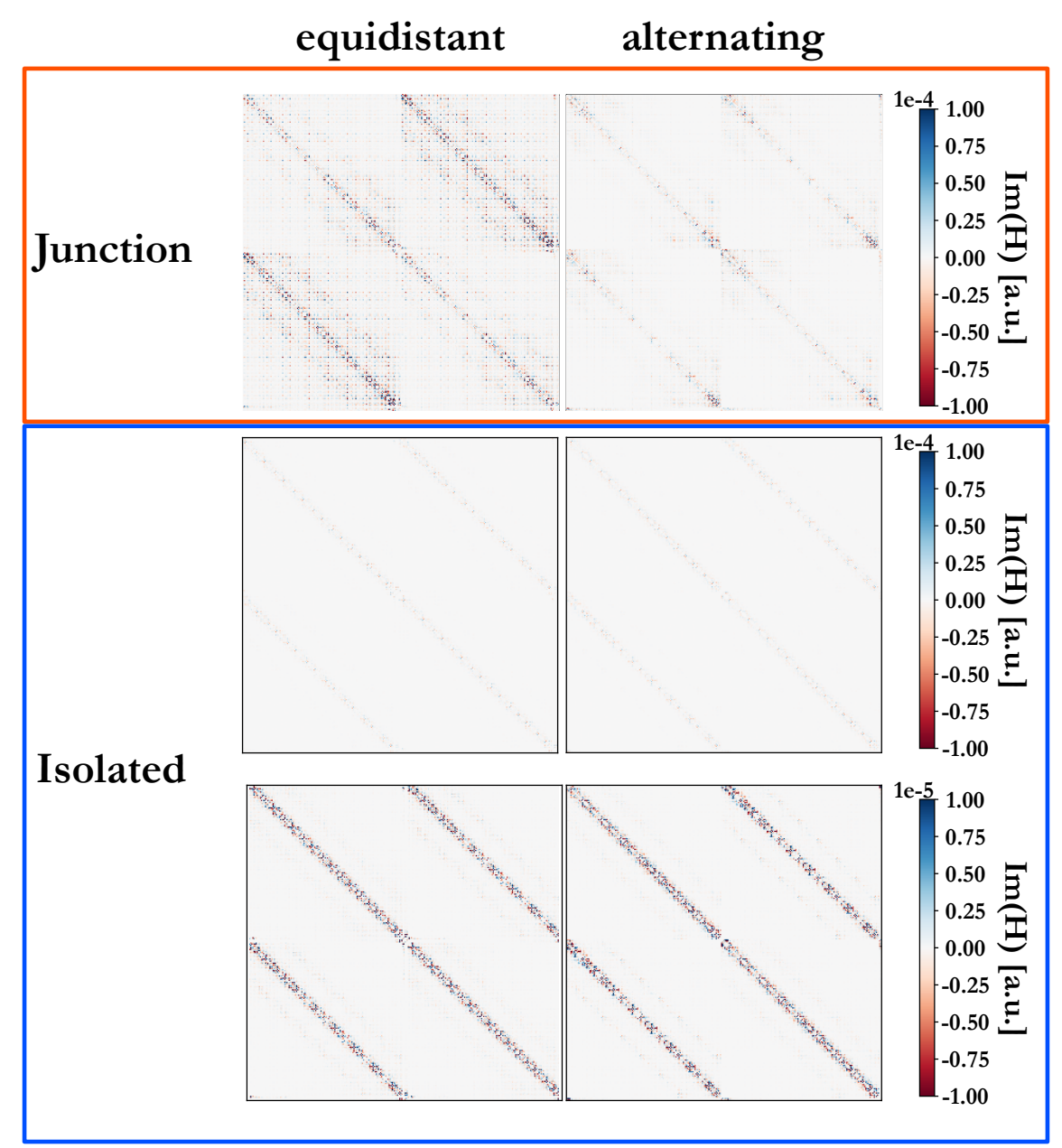

Figure 7: $\operatorname{Im}(\mathrm{H})$ of the equidistant and alternating helices in the junction (modeled by 10-atom gold clusters), plotted only for the central region consisting of the carbon and hydrogen atoms (top). For the isolated helix, values are plotted on the same scale as in the junction (middle row) and amplified by a factor of 10 (bottom row).

The transmission function and $P_{S}$ are strongly affected by the change from the equidistant to the alternating helix (see Figure 6). The transmission functions now shows a large dip in 
the vicinity of the estimated Fermi energy, and $P_{S}$ decreases massively. Looking at $\operatorname{Im}\left(\mathbf{H}_{C}\right)$ within a gold-molecule-gold junction, $\operatorname{Im}\left(\mathbf{H}_{C}\right)$ of the alternating helix (see Figure 7 ) is much smaller than $\operatorname{Im}\left(\mathbf{H}_{C}\right)$ for the equidistant helix. However, comparing $\operatorname{Im}\left(\mathbf{H}_{C}\right)$ of the isolated structures shows that for the isolated molecules, $\operatorname{Im}\left(\mathbf{H}_{C}\right)$ is quite similar. This indicates that the differences of $P_{S}$ for both bond patterns arise from differences in inherited gold SOC.

\section{Dependence of CISS on computational parameters}

\subsection{Exact-exchange admixture in the approximate exchange-cor- relation functional}

We evaluate polarization for the equidistant carbon model helix with and without 10-goldatom electrode clusters, obtained from B3LYP in terms of methodology A (see Table 1), varying the amount of Hartree-Fock exchange in the approximate exchange-correlation functional $(5 \%, 20 \%, 35 \%, 50 \%, 65 \%$, and $80 \%)$. For the isolated helix, the size of $\operatorname{Im}\left(\mathbf{H}_{C}\right)$ increases with the amount of Hartree-Fock exchange (Figure 8, bottom). However, for the $\mathrm{Au}-$ helix-Au system, the polarization of the transmitted electrons does not correlate linearly with the amount of exact exchange: From $5 \%$ to $50 \%, P_{S}$ increases, while any further increase of exact exchange leads to a decrease of $P_{S}$ (Figure 8, top). This trend is also observed in $\operatorname{Im}\left(\mathbf{H}_{C}\right)$ (Figure 8, middle). These results suggest that for the Au-helix-Au system, $\operatorname{Im}\left(\mathbf{H}_{C}\right)$, and thus $P_{S}$, is influenced by exact exchange in several ways: In addition to $\operatorname{Im}\left(\mathbf{H}_{C}\right)$ increasing intrinsically due to the larger amount of exact exchange, the alignment between metal and molecule orbitals may shift (as suggested by the changes in the transmissions shown in Figure 8), and the coupling with the gold electrode, and thus the inherited part of $\operatorname{Im}\left(\mathbf{H}_{C}\right)$, may change. By looking at the coupling blocks of $\operatorname{Re}\left(\mathbf{H}_{C}\right)$ (see Supporting Information), a clear correlation between $P_{S}$ and the coupling strength cannot be observed. The magnitude of $\operatorname{Re}\left(\mathbf{H}_{C}\right)$ of the coupling block increases from $5 \%$ to $50 \%$, 
but does not decrease significantly for $60 \%$ and $80 \%$. Thus, we cannot explain the trend in $P_{S}$ by a simple analysis of the Hamiltonian.
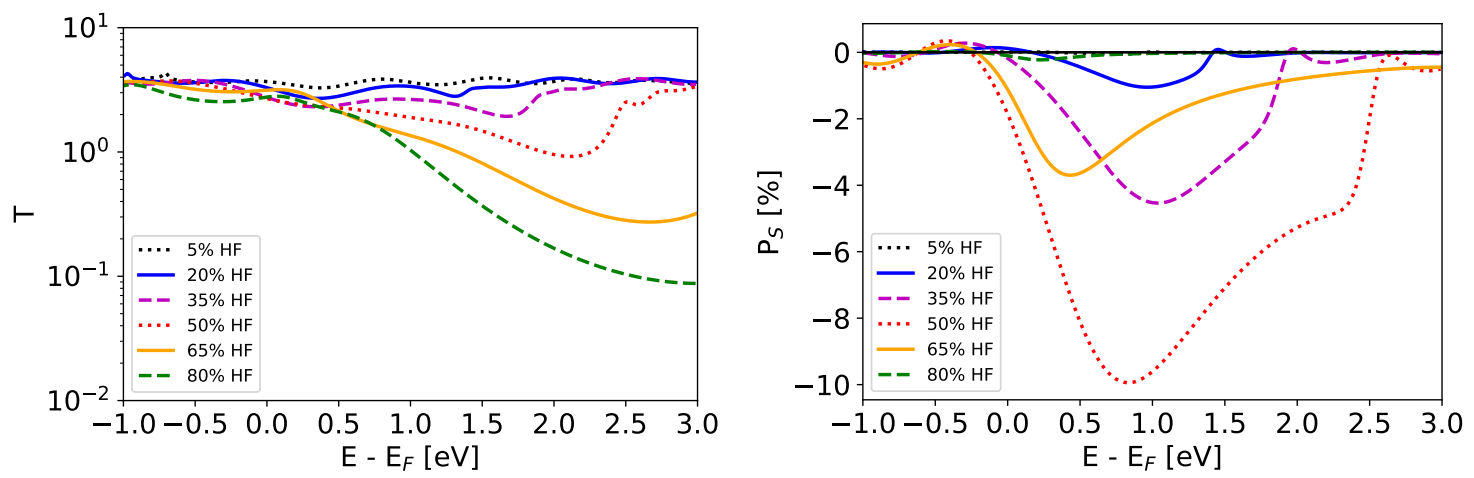

$\operatorname{Im}(\mathrm{H})$ of the carbon helix

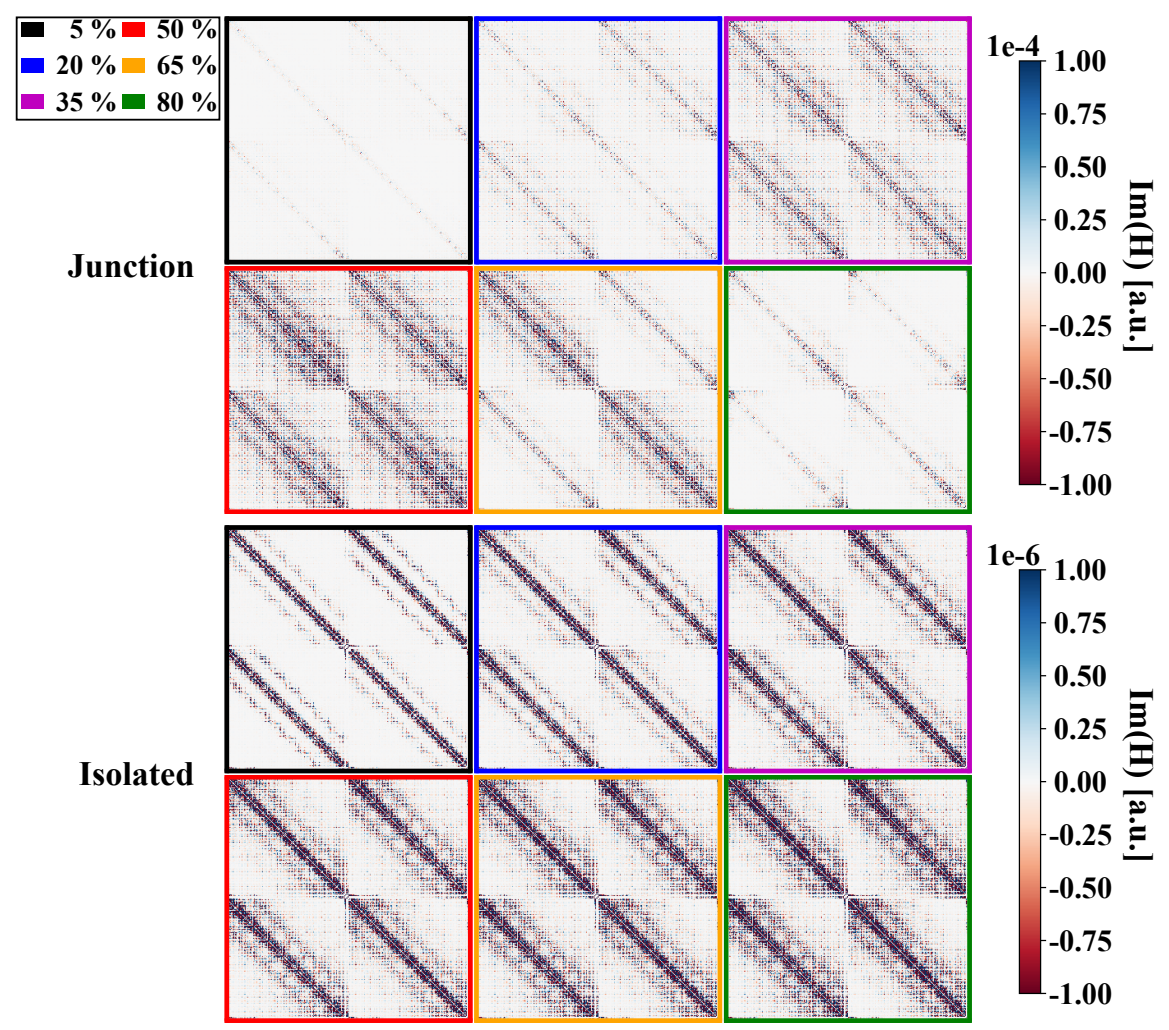

Figure 8: Calculated transmission, spin polarization of the transmitted electrons $\left(P_{S}\right)$, and $\operatorname{Im}(\mathbf{H})$ of the gold-molecule-gold junction of the equidistant helix for several amounts of exact exchange. Increasing the amount of Hartree-Fock exchange strongly influences the transmission function, leading to an opening of a gap in the transmission function. Also the calculated spin polarization of the transmitted electrons $\left(P_{S}\right)$ for the equidistant carbon helix between 10-atom gold clusters (top): For admixtures of $5 \%$ to $50 \%, P_{S}$ increases. Any further increases leads to a decrease of the calculated $P_{S}$. For the gold-molecule-gold junction, $\operatorname{Im}\left(\mathbf{H}_{C}\right)$ behaves as the calculated $P_{S}$ (middle), while for the isolated molecule, $\operatorname{Im}(\mathbf{H})$ constantly increases with the amount of exact exchange (bottom). Note the different scales for $\operatorname{Im}\left(\mathbf{H}_{C}\right)$ for the junction and the isolated molecule. 


\subsection{Two-electron terms in spin-orbit coupling}

Our results indicate that for the systems under investigation here, non-negligible $P_{S}$ can only be predicted if SOC is inherited from the electrodes. In those calculations (which employ standard implementations of SOC in electronic structure codes), SOC is only taken into account partially, i.e., only the one-electron SOC is considered for reasons of computational feasibility. However, two-electron SOC terms (spin-same orbit (SSO) and spin-other orbit (SOO)) can also contribute to the CISS effect. Taking these interactions into account could increase the intrinsic SOC of the helix and thus could lead to a significant amount of $P_{S}$ originating from the molecule itself.

To investigate the effect of the two-electron SOC terms, transmission functions were calculated based on the DFT electronic structure data obtained with methodology C (see Table 1), i.e., with the DIRAC code ${ }^{123}$ including also two-electron SOC terms. Since the self-consistent-field calculations of the $\mathrm{Au}-$ molecule- $\mathrm{Au}$ junctions could not be brought to convergence using the DIRAC code with these settings, the transmission functions were evaluated for the isolated molecules only. To do so, the electrodes for the calculation of the transmission function were chosen to be the terminal hydrogen atoms (with the same LDOS as for the metal-cluster electrodes), while the scattering region was defined as the carbon atoms. Although this workaround prevents us from quantifying the effect of the two-electron SOC terms on $P_{S}$ for the $\mathrm{Au}-$ helix-Au junction, it allows us to assess whether the intrinsic SOC of the carbon helix is significantly enhanced by including two-electron terms.

If the two-electron terms are not considered within the DFT calculation, TURBOmolE and DIRAC give the same results (Figure 9). Adding the two-electron SOC terms does not in-

crease $P_{S}$, but rather decreases it. This is consistent with calculations for the $g$-tensor. 88 The two-electron terms counteract the one-electron term, decreasing the effective SOC. Employing spin-same and spin-other orbit interactions lead to a decrease of more than $80 \%$ of $P_{S}$ compared with one-electron spin-orbit coupling only. Even if this is just shown for a 
scenario where no gold electrodes are considered, it suggests that for the development of a quantitative DFT-based method for the correct description of the CISS effect, either all SOC terms should be considered, or a careful evaluation of systematic error compensation needs to be done. Otherwise, seemingly correct values for $P_{S}$ might originate from an insufficient description of SOC.

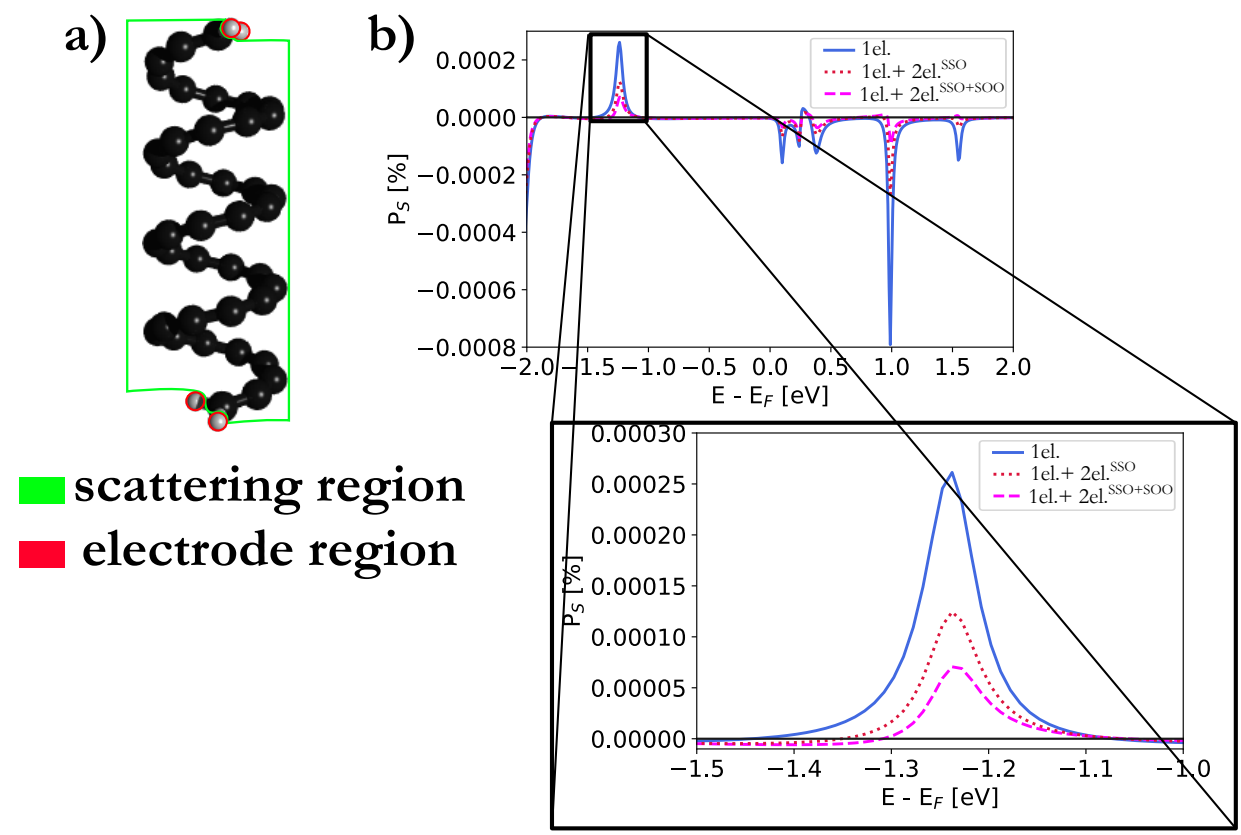

Figure 9: Calculated spin polarization of the transmitted electrons $\left(P_{S}\right)$ for the isolated equidistant carbon helix. The terminal hydrogen atoms were defined to be the electrodes, while the carbon atoms were defined to be the central region. $P_{S}$ was calculated with DIRAC without two-electron SOC (blue, 1el.), with additionally including the spin-same orbit interaction (red, 1el. $+2 \mathrm{el}^{S S O}$ ), and with all two-electron SOC terms (purple, $1 \mathrm{el} .+2 \mathrm{el}^{S S O+S O O}$ ). Adding two-electron SOC terms decreases the calculated $P_{S}$.

\section{A realistic helix: helicene}

Finally, we study a realistic system, a helicene derivative (Figure 3). Compared to other experimentally studied systems such as peptides, this molecule has a significant transmission and is therefore less prone to numerical noise. Instead of the $\mathrm{X} 2 \mathrm{C}$ method, we employ the ZORA method as implemented in the ADF program package (method D as detailed in the 
methodology section, see Table 11). We use this methodology for the helicene molecule, since two-electron SOC influences $P_{S}$, and the ZORA treatment of the SOC indirectly includes parts of the two-electron SOC due to the usage of the full Kohn-Sham potential t4 We also calculated the transmission function and $P_{S}$ for the equidistant carbon helix with method $\mathrm{D}$ as a consistency check. Both are in qualitative agreement with the results from methodology A employing Turbomole and X2C (see Supporting Information). Interestingly, the polarization calculated with ADF and ZORA is larger than the polarization calculated with Turbomole and X2C, which seems quite puzzling since ZORA contains parts of the 2electron SOC which should decrease the polarization. However, the calculation with ADF does not only describe SOC differently, but uses a different type of basis functions (SlaterType Orbitals) as well as a different basis set (ZORA-DZ).

Similar to the results by Masyluk et al ${ }^{[36}$ for a peptide, a very small value for $P_{S}$ over the whole calculated energy range is predicted with the Landauer approach. However, $P_{S}$ changes its sign after inverting the structure (see Figure 10), showing that this polarization appears to originate from the CISS effect. $\operatorname{Im}(\mathbf{H})$ of the helicene molecule within a goldmolecule-gold junction is much smaller than $\operatorname{Im}(\mathbf{H})$ of the equidistant helical molecule within a gold-molecule-gold junction (see Figure 10. This indicatesa much lower SOC transfer from gold to the helicene molecule compared with the ideal equidistant helix, which might be latter being closer to the gold surface.

Since $P_{S}$ for the perfect helical system was very sensitive to the amount of exact exchange, we check whether the polarization for helicene can be brought closer to experimental values by increasing the amount of exact exchange. This increase shifts the maximum of $P_{S}$ to higher energies (see Figure 10). This is probably due to the enlargement of the HOMOLUMO gap. However, $P_{S}$ remains by orders of magnitude too small compared with the experimental values of roughly $50 \%$. Nonetheless, one can again see the importance of the

\footnotetext{
${ }^{4}$ We had chosen methodology A, taking into account only 1-electron contributions to SOC, as our standard method for the model helices because for the alternative methodology D employed here it was not possible to extract the Hamiltonian for a perfect linear system, which was studied for comparison.
} 
Hartree-Fock exchange for the investigation of the CISS effect: Increasing it from $20 \%$ to $50 \%$ enhances $P_{S}$ by about a factor of 1.5 . Thus, for a DFT-based approach to describe the CISS effect, we would strongly suggest to not only use pure functionals, but also to check hybrid ones (and possibly more types which are beyond the scope of the present study).
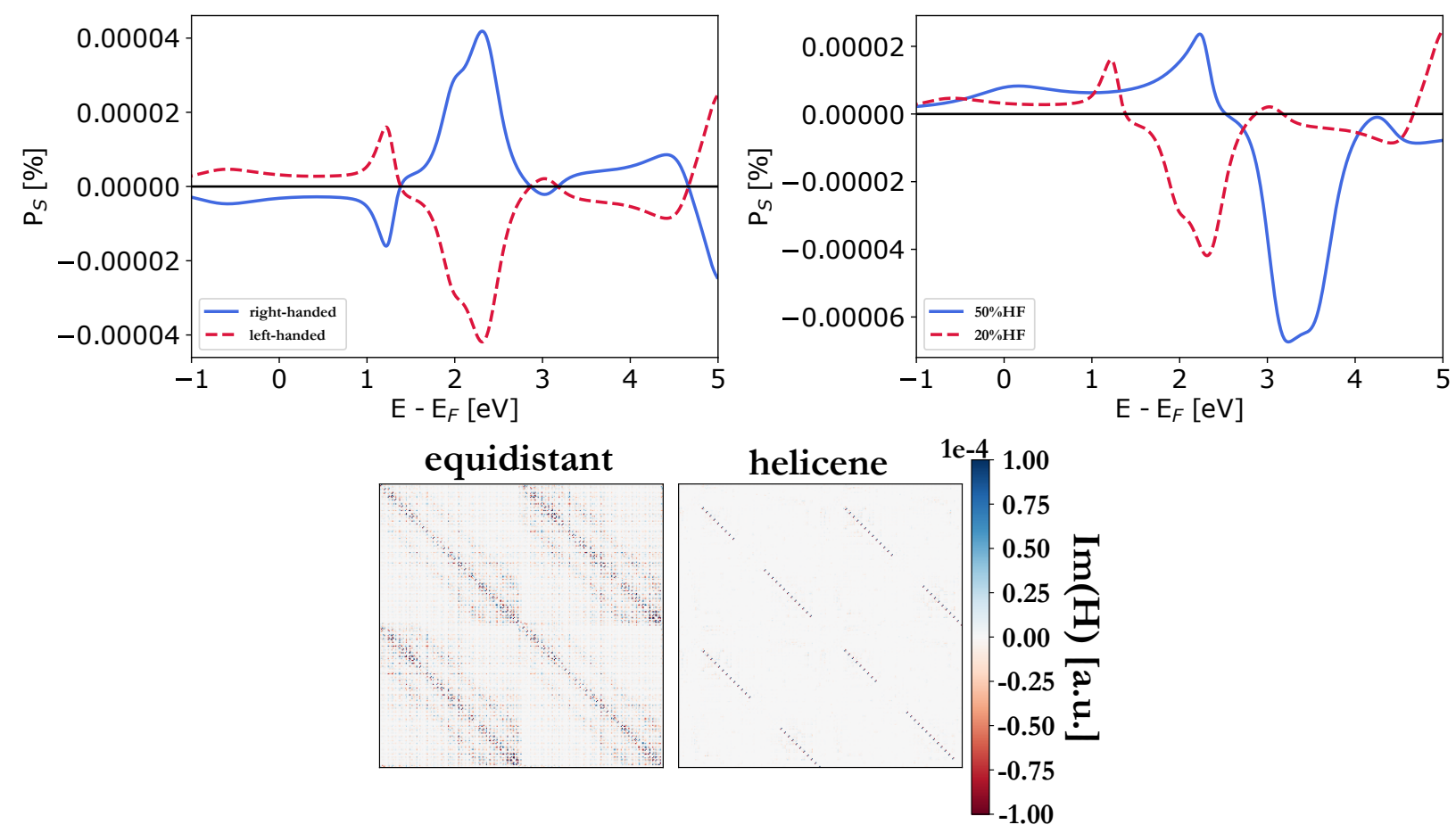

Figure 10: Calculated $P_{S}$ for the gold-helicene-gold junction using B3LYP/ZORA-DZ for the two different enantiomers of the helicene derivative and two different amounts of Hartree-Fock exchange, as well as $\operatorname{Im}(\mathbf{H})$ of the central region of the junction of the equidistant helix and the helicene. $P_{S}$ is very small compared to $P_{S}$ of the equidistant carbon helix. However, the sign of $P_{S}$ changes upon inverting the structure, indicating the CISS effect to be the origin of this polarization. Increasing the Hartree-Fock exchange increases $P_{S}$ by a factor of about 1.5, but it remains very small. $\operatorname{Im}(\mathrm{H})$ for the helicene molecule is very localized and much smaller than $\operatorname{Im}(\mathrm{H})$ of the equidistant helix, in agreement with the much larger $P_{S}$ for the equidistant helix. This indicates that for a realistic system the inherited SOC by the gold is negligible.

\section{Conclusions}

We have investigated the effect of several structural and computational parameters on the calculated magnitude of spin polarization caused by the CISS effect for artificial and realistic 
helices, based on two-component DFT combined with the Landauer-Imry-Bttiker approach. For an equidistant ideal carbon helix, SOC is mainly inherited from the gold atoms of the electrodes, and it is highly sensitive to exact exchange admixture and cluster size and shape. This is strikingly illustrated by the fact that even when SOC is only considered on the electrodes via effective core potentials and neglected on the molecule, the polarization is nearly the same as for an all-electron SOC description on the full system. When introducing bond alternation, the helix becomes less metallic and less SOC arising from the gold is present. Sensitivity to the description of exchange is also seen in the realistic helicene system (though to a lesser extent, possibly due to a larger molecule-electrode distance). Furthermore, including two-electron terms in the description of SOC decreases polarization by up to $80 \%$, even though this effect may be masked by changes in the atom-centered single-particle basis functions. We cannot exclude that the strong cluster size and shape dependence we observe is unique to the artificial helix. Yet, a possible dependence on all these parameters should be kept in mind in any future first-principles studies of CISS.

As previous work on a first-principles description of CISS,,$\sqrt[3637]{37}$ our results clearly point out the need for including further mechanisms, such as electron-phonon coupling ${ }^{138}$ or other terms resulting in leakage ${ }^{355}$ or dephasing,${ }^{2529}$ spin polarization at the interfaces, ${ }^{50}$ and an explicit description of electron correlation and nonequilibrium effects on the electronic structure ${ }^{52}$ (note that our electronic structures are obtained from a self-consistent field algorithm on finite systems in equilibrium). Yet, for these studies, our findings suggest that a careful study of the dependence on computational and structural parameters is crucial to prevent seemingly correct results due to error compensation. 


\section{Acknowledgments}

We would like to thank Markus Reiher, ETH Zurich, for helpful discussions, and the highperformance computing centre at University of Hamburg for computational resources. This work is supported by Deutsche Forschungsgemeinschaft (DFG) via the project "Structureproperty relationships for spin-orbit effects in chiral molecules" (HE 5675/4-1). V.M. acknowledges a Fellowship from Ikerbasque, the Basque Foundation for Science.

\section{References}

(1) Ray, K.; Ananthavel, S. P.; Waldeck, D. H.; Naaman, R. Asymmetric Scattering of Polarized Electrons by Organized Organic Films of Chiral Molecules. Science 1999, 238, 814-816.

(2) Naaman, R.; Paltiel, Y.; Waldeck, D. H. Chiral molecules and the electron spin. Nature Reviews 2019, 3, 250260.

(3) Mtangi, W.; Tassinari, F.; Vankayala, K.; Jentzsch, A. V.; Adelizzi, B.; Palmans, A. R. A.; Fontanesi, C.; Meijer, E. W.; Naaman, R. Control of Electrons Spin Eliminates Hydrogen Peroxide Formation During Water Splitting. J. Am. Chem. Soc. 2017, 139, $2794-2798$.

(4) Mtangi, W.; Kiran, V.; Fontanesi, C.; Naaman, R. Role of the Electron Spin Polarization in Water Splitting. J. Phys. Chem. Lett. 2015, 6, 4916-4922.

(5) Mondal, P. C.; Mtangi, W.; Fontanesi, C. ChiroSpintronics: SpinDependent Electrochemistry and Water Splitting Using Chiral Molecular Films. small methods 2018, 2, 1700313.

(6) Ghosh, K. B.; Zhang, W.; Tassinari, F.; Mastai, Y.; Lidor-Shalev, O.; Naaman, R.; Möllers, P.; Nürenberg, D.; Zacharias, H.; Wei, J.; Wierzbinski, E.; Waldeck, D. H. 
Controlling Chemical Selectivity in Electrocatalysis with Chiral CuO-Coated Electrodes. J. Chem. Phys. C 2019, 123, 3024-3031.

(7) Banerjee-Ghosh, K.; Ben Dor, O.; Tassinari, F.; Capua, E.; Yochelis, S.; Capua, A.; Yang, S.-H.; Parkin, S. S. P.; Sarkar, S.; Kronik, L.; Baczewski, L. T.; Naaman, R.; Paltiel, Y. Separation of enantiomers by their enantiospecific interaction with achiral magnetic substrates. Science 2018, 360, 1331-1334.

(8) Brandt, J. R.; Salerno, F.; Fuchter, M. J. The added value of small-molecule chirality in technological applications. Nat. Rev. Chem. 2017, 1, 0045.

(9) Michaeli, K.; Kantor-Uriel, N.; Naaman, R.; Waldeck, D. H. The electron's spin and molecular chirality how are they related and how do they affect life processes? Chem. Soc. Rev. 2016, 45, 6478-6487.

(10) Aragones, A. C.; Medina, E.; Ferrer-Huerta, M.; Gimeno, N.; Teixido, M.; Palma, J. L.; Tao, N.; Ugalde, J. M.; Giralt, E.; Diez-Perez, I.; Mujica, V. Measuring the SpinPolarization Power of a Single Chiral Molecule. small 2017, 13, 1602519.

(11) Abendroth, J. M.; Cheung, K. M.; Stemer, D. M.; Hadri, M. S. E.; Zhao, C.; Fullerton, E. E.; Weiss, P. S. Spin-Dependent Ionization of Chiral Molecular Films. J. Am. Chem. Soc. 2019, 141, 3863-3874.

(12) Gazzotti, M.; Arnaboldi, S.; Grecchi, S.; Giovanardi, R.; Cannio, M.; Pasquali, L.; Giacomino, A.; Abollino, O.; Fontanesi, C. Spin-dependent electrochemistry: Enantioselectivity driven by chiral-induced spin selectivity effect. Electrochimica Acta 2018, 286, 271-278.

(13) Santos, J. I.; Rivilla, I.; o P. Cossio, F.; Matxain, J. M.; Grzelczak, M.; Mazinani, S. K. S.; Ugalde, J. M.; Mujica, V. Chirality-Induced Electron Spin Polarization and Enantiospecific Response in Solid-State Cross-Polarization Nuclear Magnetic Resonance. ACS Nano 2018, 12, 11426-11433. 
(14) Alpern, H.; Yavilberg, K.; Dvir, T.; Sukenik, N.; Klang, M.; Yochelis, S.; Cohen, H.; Grosfeld, E.; Steinberg, H.; Paltiel, Y.; Millo, O. Magnetic-related States and Order Parameter Induced in a Conventional Superconductor by Nonmagnetic Chiral Molecules. Nano Lett. 2019, 19, 5167-5175.

(15) Xie, Z.; Markus, T. Z.; Cohen, S. R.; Vager, Z.; Gutierrez, R.; Naaman, R. Spin Specific Electron Conduction through DNA Oligomers. Nano Lett. 2011, 11, 4652-4655.

(16) Zwang, T. J.; Hürlimann, S.; Hill, M. G.; Barton, J. K. Helix-Dependent Spin Filtering through the DNA Duplex. J. Am. Chem. Soc. 2016, 138, 15551-15554.

(17) Göhler, B.; Hamelbeck, V.; Markus, T. Z.; Kettner, M.; Hanne, G. F.; Vager, Z.; Naaman, R.; Zacharias, H. Spin selectivity in electron transmission through self-assembled monolayers of double-stranded DNA. Science 2011, 331, 894-897.

(18) Kettner, M.; Göhler, B.; Zacharias, H.; Mishra, D.; Kiran, V.; Naaman, R.; Fontanesi, C.; Waldeck, D. H.; Sek, S.; Pawlowski, J.; Juhaniewicz, J. Spin Filtering in Electron Transport Through Chiral Oligopeptides. J. Phys. Chem. C 2015, $119,14542-14547$.

(19) Mishra, D.; Markus, T. Z.; Naaman, R.; Kettner, M.; Göhler, B.; Zacharias, H.; Friedman, N.; Sheves, M.; Fontanesi, C. Spin-dependent electron transmission through bacteriorhodopsin embedded in purple membrane. Proc. Natl. Acad. Sci. 2013, 110, $14872-14876$.

(20) Kettner, M.; Maslyuk, V. V.; Nrenberg, D.; Seibel, J.; Gutierrez, R.; Cuniberti, G.; Ernst, K.-H.; Zacharias, H. Chirality-Dependent Electron Spin Filtering by Molecular Monolayers of Helicenes. J. Phys. Chem. Lett. 2018, 9, 2025-2030.

(21) Kiran, V.; Mathew, S. P.; Cohen, S. R.; Delgado, I. H.; Lacour, J.; Naaman, R. Helicenes - A New Class of Organic Spin Filter. Adv. Mater. 2016, 28, 1957-1962. 
(22) Winkler, R., Ed. Spin-Orbit Coupling Effects in Two-Dimensional Electron and Hole Systems; 2003.

(23) Forster, F.; Bendounan, A.; Reinert, F.; Grigoryan, V. G.; Springborg, M. The Shockley-type surface state on $\mathrm{Ar}$ covered $\mathrm{Au}(111)$ : High resolution photoemission results and the description by slab-layer DFT calculations. Surf. Sci. 2007, 601, 55955604.

(24) Petersen, L.; Hedegård, P. A simple tight-binding model of spin-orbit splitting of sp-derived surface states. Surf. Sci. 2000, 459, 4956.

(25) Guo, A.-M.; Sun, Q.-F. Spin-dependent electron transport in protein-like single-helical molecules. Proc. Natl. Acad. Sci. 2014, 111, 11658-11662.

(26) Skourtis, S. S.; Beratan, D. N.; Naaman, R.; Nitzan, A.; Waldeck, D. H. Chiral Control of Electron Transmission through Molecules. Phys. Rev. Lett. 2008, 101, 238103.

(27) Pan, T.-R.; Guo, A.-M.; Sun, Q.-F. Effect of gate voltage on spin transport along $\alpha$-helical protein. Phys. Rev. B 2015, 92, 115418.

(28) Sierra, M. A.; Sanchez, D.; Gutierrez, R.; Cuniberti, G.; Dominguez-Adame, F.; Diaz, E. Spin-Polarized Electron Transmission in DNA-Like Systems. Biomolecules 2020, 10, 49 .

(29) Guo, A.-M.; feng Sun, Q. Spin-Selective Transport of Electrons in DNA Double Helix. Phys Rev. Lett. 2012, 108, 218102.

(30) Gutierrez, R.; Diaz, E.; Naaman, R.; Cuniberti, G. Spin-selective transport through helical molecular systems. Phys. Rev. B 2012, 85, 081404.

(31) Gutierrez, R.; Diaz, E.; Gaul, C.; Brumme, T.; Dominguez-Adame, F.; Cuniberti, G. Modeling Spin Transport in Helical Fields: Derivation of an Effective Low-Dimensional Hamiltonian. J. Phys. Chem. C 2013, 117, 22276-22284. 
(32) Diaz, E.; Francisco,; Dominguez-Adame,; Gutierrez, R.; Cuniberti, G.; Mujica, V. Thermal Decoherence and Disorder Effects on Chiral-Induced Spin Selectivity. J. Phys. Chem. Lett. 2008, 9, 5753-5758.

(33) Varela, S.; Mujica, V.; Medina, E. Effective spinorbit couplings in an analytical tightbinding model of DNA: spin filtering and chiral spin transport. Phys. Rev. B $\mathbf{2 0 1 6}$, 93, 155436 .

(34) Varela, S.; Mujica, V.; Medina, E. Spin-orbit Coupling Modulation in DNA by Mechanical Deformations. Chimia 2018, 72, 411-417.

(35) Geyer, M.; Gutierrez, R.; Mujica, V.; Cuniberti, G. Chirality-Induced Spin Selectivity in a Coarse-Grained Tight-Binding Model for Helicene. J. Phys. Chem. C 2019, just accepted.

(36) Maslyuk, V. V.; Gutierrez, R.; Dianat, A.; Mujica, V.; Cuniberti, G. Enhanced Magnetoresistance in Chiral Molecular Junctions. J. Phys. Chem. Lett. 2018, 9, 5453-5459.

(37) Zöllner, M. S.; Varela, S.; Medina, E.; Mujica, V.; Herrmann, C. Insight into the Origin of Chiral-Induced Spin Selectivity from a Symmetry Analysis of Electronic Transmission. J. Chem. Theory Comput. 2020, 16, 2914-2929, ChemRxiv, DOI: 10.26434/chemrxiv.8325248.v2.

(38) Naaman, R.; Waldeck, D. H. Annu. Rev. Phys. Chem. 2015, 66, 263-281.

(39) Evers, F.; Korytar, R.; Tewari, S.; van Ruitenbeek, J. M. Advances and challenges in single-molecule electron transport. 2019, arXiv:1906.10449v1.

(40) Büttiker, M. Role of quantum coherence in series resistors. Phys. Rev. B 1986, 333, 3020-3026.

(41) Cattena, C. J.; Fernandez-Alcazar, L. J.; Bustos-Marun, R. A.; Nozaki, D.; Pastawski, H. M. Generalized multi-terminal decoherent transport: recursive algo- 
rithms and applications to SASER and giant magnetoresistance. J. Phys.: Condens. Matter 2014, 26, 345304.

(42) Nozaki, D.; Bustos-Marun, R.; Cattena, C. J.; Cuniberti, G.; Pastawski, H. M. An efficient coarse-grained approach for the electron transport through large molecular systems under dephasing environment. Eur. Phys. J. B (2016) 89: 102 2016, 89, 102.

(43) Nozaki, D.; da Rocha, C. G.; Pastawski, H. M.; Cuniberti, G. Disorder and dephasing effects on electron transport through conjugated molecular wires in molecular junctions. Phys. Rev. B 2012, 85, 155327.

(44) Bandyopadhyay, M.; Segal, D. Quantum heat transfer in harmonic chains with selfconsistent reservoirs: Exact numerical simulations. Phys. Rev. E 2011, 84, 011151.

(45) Matityahu, A.; Utsumi, Y.; Aharony, A.; Entin-Wohlman, O.; Balseiro, C. A. Spindependent transport through a chiral molecule in the presence of spin-orbit interaction and nonunitary effects. Phys. Rev. B 2016, 93, 075407.

(46) Yang, X.; van der Wal, C. H.; van Wees, B. J. Detecting chirality in two-terminal electronic devices. 2019, arXiv:1912.09085.

(47) Yang, X.; van der Wal, C. H.; van Wees, B. J. Spin-dependent electron transmission model for chiral molecules in mesoscopic devices. Phys. Rev. B 2019, 99, 024418.

(48) Yang, X.; van der Wal, C. H.; van Wees, B. J. Reply to Comment on "Spin-dependent electron transmission model for chiral molecules in mesoscopic devices". Phys. Rev. B 2020, 101, 026404.

(49) Naaman, R.; Waldeck, D. H. Comment on "Spin-dependent electron transmission model for chiral molecules in mesoscopic devices". Phys. Rev. B 2020, 101, 026403.

(50) Dalum, S.; Hedegård, P. Theory of Chiral Induced Spin Selectivity. Nano Lett. 2019, $19,5253-5259$. 
(51) Michaeli, K.; Beratan, D. N.; Waldeck, D. H.; Naaman, R. Voltage-induced long-range coherent electron transfer through organic molecules. Proc. Nat. Acad. Sci. 2019, 116, $5931-5936$.

(52) Fransson, J. Chirality-Induced Spin Selectivity: The Role of Electron Correlations. J. Phys. Chem. Lett. 2019, 10, 7126-7132.

(53) Cohen, A. J.; Mori-Sanchez, P.; Yang, W. Challenges for Density Functional Theory. Chem. Rev. 2012, 112, 289-320.

(54) Verma, P.; G.Truhlar, D. Status and Challenges of Density Functional Theory. Trends Chem. 2020, 2, 302-318.

(55) Cramer, C. J.; Truhlar, D. G. Density functional theory for transition metals and transition metal chemistry. Phys. Chem. Chem. Phys. 2009, 11, 10757-10816.

(56) Ke, S.-H.; Baranger, H. U.; Yang, W. Role of the exchange-correlation potential in ab initio electron transport calculations. J. Phys. Chem. 2007, 126, 201102.

(57) Koentopp, M.; Chang, C.; Burke, K.; Car, R. Density functional calculations of nanoscale conductance. J. Phys.: Condens. Matter 2008, 20, 083203.

(58) Koentopp, M.; Burke, K.; Evers, F. Zero-bias molecular electronics: Exchangecorrelation corrections to Landauer's formula. Phys. Rev. B 2006, 73, 121403(R).

(59) Thoss, M.; Evers, F. Perspective: Theory of quantum transport in molecular junctions. J. Chem. Phys. 2018, 148, 030901.

(60) Mujica, V.; Kemp, M.; Ratner, M. A. Electron conduction in molecular wires. I. A scattering formalism. J. Chem. Phys. 1994, 101, 6849-6855.

(61) Lindsay, S. M.; Ratner, M. A. Molecular Transport Junctions: Clearing Mists. Adv. Mater. 2007, 19, 23-31. 
(62) Pernot, P. The parameter uncertainty inflation fallacy. J. Chem. Phys. 2017, 147, 104102.

(63) Pernot, P.; Savin, A. Probabilistic performance estimators for computational chemistry methods: The empirical cumulative distribution function of absolute errors. $J$. Chem. Phys. 2018, 148, 241707.

(64) Proppe, J.; Reiher, M. Reliable Estimation of Prediction Uncertainty for Physicochemical Property Models. J. Chem. Theory Comput. 2017, 13, 3297-3317.

(65) Simm, G. N.; Reiher, M. Systematic Error Estimation for Chemical Reaction Energies. J. Chem. Theory Comput. 2016, 12, 2762-2773.

(66) Simm, G.; Proppe, J.; Reiher, M. Error Assessment of Computational Models in Chemistry. Chimia 2017, 71, 202-208.

(67) Weymuth, T.; Proppe, J.; Reiher, M. Statistical Analysis of Semiclassical Dispersion Corrections. J. Chem. Theory Comput. 2018, 14, 2480-2494.

(68) Mortensen, J. J.; Kaasbjerg, K.; Frederiksen, S. L.; Nørskov, J. K.; Jacobsen, J. P. S. K. W. Bayesian error estimation in density-functional theory. Phys. Rev. Lett. 2005, 95, 216401.

(69) Wellendorff, J.; Lundgaard, K. T.; Mogelhoj, A.; Petzold, V.; Landis, D. D.; Norskov, J. K.; Bligaard, T.; Jacobsen, K. W. Density functionals for surface science: Exchange-correlation model development with Bayesian error estimation. Phys. Rev. B: Condens. Matter Mater. Phys. 2012, 85, 235149.

(70) Wellendorff, J.; Lundgaard, K. T.; Jacobsen, K. W.; Bligaard, T. mBEEF: an accurate semi-local Bayesian error estimation density functional. J. Chem. Phys. 2014, 140, 144107. 
(71) Bergmann, T. G.; Welzel, M. O.; Jacob, C. R. Towards theoretical spectroscopy with error bars: systematic quantification of the structural sensitivity of calculated spectra. Chem. Sci. 2020, 11, 1862-1877.

(72) Oung, S. W.; Rudolph, J.; Jacob, C. R. Uncertainty quantification in theoretical spectroscopy: The structural sensitivity of Xray emission spectra. Int. J. Quantum Chem. 2018, 118, e25458.

(73) Duan, C.; Janet, J. P.; Liu, F.; Nandy, A.; ; Kulik, H. J. Learning from Failure: Predicting Electronic Structure Calculation Outcomes with Machine Learning Models. J. Chem. Theory Comput. 2019, 15, 2331-2345.

(74) Liu, J. P. J.; Duan, A. N. C.; Yang, T.; Lin, S.; Kulik, H. J. Designing in the Face of Uncertainty: Exploiting Electronic Structure and Machine Learning Models for Discovery in Inorganic Chemistry. Inorg. Chem. 2019, 58, 10592-10606.

(75) Aspuru-Guzik, A.; Lindh, R.; Reiher, M. The Matter Simulation (R)evolution. ACS Cent. Sci. 2018, 4, 144-152.

(76) Dral, P. O. Quantum Chemistry in the Age of Machine Learning. J. Phys. Chem. Lett. 2020, 11, 2336-2347.

(77) Cova, T. F. G. G.; Pais, A. A. C. C. Deep Learning for Deep Chemistry: Optimizing the Prediction of Chemical Patterns. Front. Chem. 2019, 7.

(78) Mater, A. C.; Coote, M. L. Deep Learning in Chemistry. J. Chem. Inf. Model. 2019, $59,2545-2559$.

(79) Armbruster, M. K.; Weigend, F.; Wüllen, C.; Klopper, W. Self-consistent treatment of spinorbit interactions with efficient HartreeFock and density functional methods. Phys. Chem. Chem. Phys. 2008, 10, 1748-1756. 
(80) van Wüllen, C. A Quasirelativistic Two-component Density Functional and HartreeFock Program. Z. Phys. Chem. 2010, 224, 413.

(81) Rai, D.; Galperin, M. Electrically Driven Spin Currents in DNA. J. Phys. Chem. C 2013, 117, 13730-13737.

(82) Kuemmeth, F.; Ilani, S.; Ralph, D. C.; McEuen, P. L. Coupling of spin and orbital motion of electrons in carbon nanotubes. Nature 2008, 452, 448-452.

(83) G. A. Steele, F. P.; Laird, E. A.; Jol, J. M.; Meerwaldt, H. B.; Kouwenhoven, L. P. Large spin-orbit coupling in carbon nanotubes. Nature Commun. 2013, 4, 1573.

(84) Berche, B.; Mireles, F.; Medina, E. Rashba spin-orbit interaction enhanced by graphene in-plane deformations. Condens. Matter Phys. 2017, 20, 13702.

(85) Huertas-Hernando, D.; Guinea, F.; Brataas, A. Spin-orbit coupling in curved graphene, fullerenes, nanotubes, and nanotube caps. Phys. Rev. B 2006, 74, 155426.

(86) Min, H.; Hill, J. E.; Sinitsyn, N. A.; Sahu, B. R.; Kleinman, L.; MacDonald, A. H. Intrinsic and rashba spin-orbit interactions in graphene sheets. Phys. Rev. B 2006, $74,165310$.

(87) Reiher, M.; Wolf, A. Relativistic Quantum Chemistry, 2nd ed.; Wiley-VCH: Weinheim, 2015.

(88) Neese, F. Efficient and accurate approximations to the molecular spin-orbit coupling operator and their use in molecular g-tensor calculations. J. Chem. Phys. 2005, 122, 034107.

(89) Cuevas, J. C.; Scheer, E. In Molecular Electronics: An Introduction to Theory and Experiment, 2nd ed.; Reed, M., Ed.; World Scientific Series in Nanotechnology and Nanoscience; World Scientific: Singapore, 2017; Vol. 1. 
(90) Zotti, L. A.; Cuevas, J. C. Electron Transport Through Homopeptides: Are They Really Good Conductors? ACS Omega 2018, 3, 3778-3785.

(91) Sepunaru, L.; Refaely-Abramson, S.; Lovrincic, R.; Gavrilov, Y.; Agrawal, P.; Levy, Y.; Kronik, L.; Pecht, I.; Sheves, M.; Cahen, D. Electronic Transport via Homopeptides: The Role of Side Chains and Secondary Structure. J. Am. Chem. Soc. 2015, 137, $9617-9626$.

(92) Xiang, L.; Palma, J. L.; Bruot, C.; Mujica, V.; Ratner, M. A.; Tao, N. Intermediate tunnelling-hopping regime in DNA charge transport. Nature Chem. 2015, 7, 221-226.

(93) Caroli, C.; Combescot, R.; Nozieres, P.; Saint-James, D. Direct calculation of the tunneling current. J. Phys. C: Solid State Phys. 1971, 4, 916-929.

(94) Wilhelm, J.; Walz, M.; Evers, F. Ab initio spin-flip conductance of hydrogenated graphene nanoribbons: Spin-orbit interaction and scattering with local impurity spins. Phys. Rev. B 2015, 92, 014405.

(95) Bhattacharya, S.; Akande, A.; Sanvito, S. Spin transport properties of triarylaminebased nanowires. Chem. Commun. 2014, 50, 6626-6629.

(96) Reckien, W.; Eggers, M.; Bredow, T. Theoretical study of the adsorption of benzene on coinage metals. Beilstein J. Org. Chem. 2014, 10, 1775-1784.

(97) Ahlrichs et al., R. http://www.cosmologic.de/turbomole.html.

(98) Treutler, O.; Ahlrichs, R. Efficient molecular numerical integration schemes. J. Chem. Phys. 1995, 102, 346-354.

(99) Von Arnim, M.; Ahlrichs, R. Performance of parallel TURBOMOLE for density functional calculations. J. Comput. Chem. 1998, 19, 1746-1757. 
(100) Balasubramani, S. G. et al. TURBOMOLE: Modular program suite for ab initio quantum-chemical and condensed-matter simulations. J. Chem. Phys. 2020, 152, 184107.

(101) Perdew, J. P.; Burke, K.; Wang, Y. Generalized gradient approximation for the exchange-correlation hole of a many-electron system. Phys. Rev. B 1996, 54, 1653316539.

(102) Perdew, J. P.; Burke, K.; Ernzerhof, M. Generalized Gradient Approximation Made Simple. Phys. Rev. Lett. 1996, 77, 3865-3868.

(103) Schäfer, A.; Huber, C.; Ahlrichs, R. Fully optimized contracted Gaussian basis sets of triple zeta valence quality for atoms Li to Kr. J. Chem. Phys. 1994, 100, 5829-5835.

(104) Weigend, F.; Ahlrichs, R. Balanced basis sets of split valence, triple zeta valence and quadruple zeta valence quality for H to Rn: Design and assessment of accuracy. Phys. Chem. Chem. Phys. 2005, 7, 3297-3305.

(105) Grimme, S.; Antony, J.; Ehrlich, S.; Krieg, H. A consistent and accurate ab initio parametrization of density functional dispersion correction (DFT-D) for the 94 elements H-Pu. J. Chem. Phys. 2010, 132, 154104.

(106) Grimme, S.; Ehrlich, S.; Goerigk, L. Effect of the damping function in dispersion corrected density functional theory. J. Comput. Chem. 2011, 32, 1456-1465.

(107) Eichkorn, K.; Treutler, O.; Öhm, H.; Häser, M.; Ahlrichs, R. Auxiliary basis sets to approximate Coulomb potentials. Chem. Phys. Lett. 1995, 240, $283-290$.

(108) Eichkorn, K.; Weigend, F.; Treutler, O.; Ahlrichs, R. Auxiliary basis sets for main row atoms and transition metals and their use to approximate Coulomb potentials. Theor. Chem. Acc. 1997, 97, 119-124. 
(109) Weigend, F. Accurate Coulomb-fitting basis sets for H to Rn. Phys. Chem. Chem. Phys. 2006, 8, 1057-1065.

(110) Dyall, K. G. Interfacing relativistic and nonrelativistic methods. I. Normalized elimination of the small component in the modified Dirac equation. J. Chem. Phys. 1997, 106, 9618-9626.

(111) Kutzelnigg, W.; Liu, W. Quasirelativistic theory equivalent to fully relativistic theory. J. Chem. Phys. 2005, 123, 241102.

(112) Pollak, P.; Weigend, F. Segmented Contracted Error-Consistent Basis Sets of Doubleand Triple-Zeta Valence Quality for One- and Two-Component Relativistic AllElectron Calculations. J. Chem. Theory Comput. 2017, 13, 3696-3705.

(113) van Lenthe, E.; Baerends, E. J.; Snijders, J. G. Relativistic regular twocomponent Hamiltonians. J. Chem. Phys. 1993, 99, 4597.

(114) van Lenthe, E.; Baerends, E. J.; Snijders, J. G. Relativistic total energy using regular approximations. J. Chem. Phys. 1994, 101, 9783.

(115) van Lenthe, E.; Ehlers, A.; Baerends, E.-J. Geometry optimizations in the zero order regular approximation for relativistic effects. J. Chem. Phys. 1999, 110, 8943.

(116) van Lenthe, E.; Snijders, J. G.; Baerends, E. J. The zeroorder regular approximation for relativistic effects: The effect of spinorbit coupling in closed shell molecules. $J$. Chem. Phys. 1996, 105, 6505.

(117) van Lenthe, E.; van Leeuwen, R.; Baerends, E. J.; Snijders, J. G. Relativistic regular two-component Hamiltonians. Int. J. Quantum Chem. 1996, 57, 281.

(118) Van Lenthe, E.; Baerends, E. J. Optimized Slater-type basis sets for the elements 1118. J. Comput. Chem. 2003, 24, 1142. 
(119) Baerends, E. J. et al. ADF2014, SCM, Theoretical Chemistry, Vrije Universiteit, Amsterdam, The Netherlands, https://www.scm.com.

(120) te Velde, G.; Bickelhaupt, F. M.; Baerends, E. J.; Fonseca Guerra, C.; van Gisbergen, S. J. A.; Snijders, J. G.; Ziegler, T. Chemistry with ADF. J. Comput. Chem. 2001, 22, 931.

(121) Fonseca Guerra, C.; Snijders, J. G.; te Velde, G.; Baerends, E. J. Towards an order-N DFT method. Theor. Chem. Acc. 1998, 99, 391.

(122) Weigend, F.; Baldes, A. Segmented contracted basis sets for one- and two-component DiracFock effective core potentials. J. Chem. Phys. 2010, 133, 174102.

(123) Saue, T. et al. The DIRAC code for relativistic molecular calculations. 2020.

(124) Dyall, K. G. Relativistic double-zeta, triple-zeta, and quadruple-zeta basis sets for the light elements HAr. Theor. Chem. Acc. 2016, 135, 128.

(125) Becke, A. D. Density-functional thermochemistry. III. The role of exact exchange,. J. Chem. Phys. 1993, 98, 5648-5652.

(126) Lee, C.; Yang, W.; Parr, R. G. Development of the Colle-Salvetti correlation-energy formula into a functional of the electron density,. Phys. Rev. B 1988, 37, 785-789.

(127) Becke, A. D. Density-functional exchange-energy approximation with correct asymptotic behavior. Phys. Rev. A 1988, 38, 3098-3100.

(128) Deffner, M.; Groß, L.; Steenbock, T.; Voigt, B. A.; Zöllner, M. S.; Solomon, G. C.; Herrmann, C. 2008-2020; ARTAIOs - a postprocessing framework for electronic structure calculations, https://www.chemie.unihamburg.de/institute/ac/arbeitsgruppen/herrmann/software/artaios.html.

(129) Verzijl, C. J. O.; Seldenthuis, J. S.; Thijssen, J. M. Applicability of the wide-band limit in DFT-based molecular transport calculations. J. Chem. Phys. 2013, 138, 094102. 
(130) Papaconstantopoulos, D. A. Handbook of the Band Structure of Elemental Solids; Plenum: New York, 1986.

(131) Ke, S.-H.; Baranger, H. U.; Yang, W. Electron transport through molecules: Selfconsistent and non-self-consistent approaches . Phys. Rev. B 2004, 70, 085410.

(132) Ke, S.-H.; Baranger, H. U.; Yang, W. Models of electrodes and contacts in molecular electronics. J. Chem. Phys. 2005, 123, 114701.

(133) Lopez, A.; Colmenarez, L.; Peralta, M.; Mireles, F.; Medina, E. Proximity-induced spin-orbit effects in graphene on Au. Phys. Rev. B 2019, 99, 085411.

(134) Marchenko, D.; Varykhalov, A.; Scholz, M. R.; Bihlmayer, G.; Rashba, E. I.; Rybkin, A.; Shikin, A. M.; Rader, O. Nature Commun. 2012, 3, 1232.

(135) Mishra, S.; Mondal, A. K.; Pal, S.; Das, T. K.; Smolinsky, E. Z. B.; Siligardi, G.; Naaman, R. Length-Dependent Electron Spin Polarization in Oligopeptides and DNA. J. Phys. Chem. C 2020, 124, 10776-10782.

(136) Guo, A.-M.; Diaz, E.; Gaul, C.; Gutierrez, R.; Dominguez-Adame, F.; Cuniberti, G.; feng $\mathrm{Su}, \mathrm{Q}$. Contact effects in spin transport along double-helical molecules. Phys. Rev. B 2014, 89, 205434.

(137) Guo, A.-M.; Pan, T.-R.; Fang, T.-F.; Xie, X. C.; Sun, Q.-F. Spin selectivity effect in achiral molecular systems. Phys. Rev. B 2016, 94, 165409.

(138) Wu, Y.; Miao, G.; Subotnik, J. Chemical Reaction Rates for Systems with Spin-Orbit Coupling and an Odd Number of Electrons: Does Berry's Phase Lead to Meaningful Spin-Dependent Nuclear Dynamics for a Two State Crossing? 2020; arXiv 2005.10424. 


\section{Graphical TOC Entry}

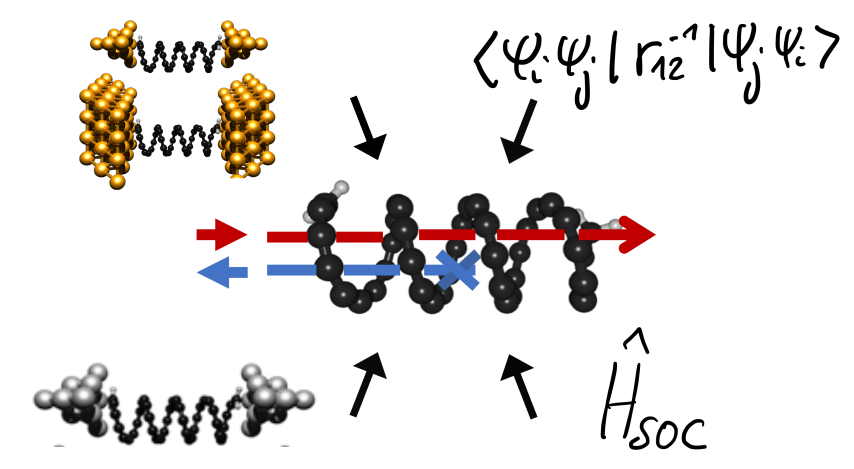

